\title{
Thermoplastic Cellulose-Based Compound for Additive Manufacturing
}

\author{
Kirsi Immonen *, Pia Willberg-Keyriläinen, Jarmo Ropponen, Asta Nurmela, Sini Metsä-Kortelainen (D), \\ Otto-Ville Kaukoniemi and Heli Kangas (1)
}

check for

updates

Citation: Immonen, K.;

Willberg-Keyriläinen, P.; Ropponen, J.;

Nurmela, A.; Metsä-Kortelainen, S.;

Kaukoniemi, O.-V.; Kangas, H.

Thermoplastic Cellulose-Based

Compound for Additive

Manufacturing. Molecules 2021, 26,

1701. https://doi.org/10.3390/

molecules26061701

Academic Editor: Patrick M. Martin

Received: 24 February 2021

Accepted: 16 March 2021

Published: 18 March 2021

Publisher's Note: MDPI stays neutral with regard to jurisdictional claims in published maps and institutional affiliations.

Copyright: (C) 2021 by the authors. Licensee MDPI, Basel, Switzerland. This article is an open access article distributed under the terms and conditions of the Creative Commons Attribution (CC BY) license (https:// creativecommons.org/licenses/by/ $4.0 /)$.
VTT Technical Research Centre of Finland Ltd., Tietotie 4E, P.O. Box 1000, FI-02044 VTT, FI-02150 Espoo, Finland; pia.willberg-keyrilainen@vtt.fi (P.W.-K.); jarmo.ropponen@vtt.fi (J.R.); asta.nurmela@vtt.fi (A.N.); sini.metsa-kortelainen@vtt.fi (S.M.-K.); otto-ville.kaukoniemi@vtt.fi (O.-V.K.); heli.kangas@vtt.fi (H.K.)

* Correspondence: kirsi.immonen@vtt.fi; Tel.: +358-40-5185351

\begin{abstract}
The increasing environmental awareness is driving towards novel sustainable highperformance materials applicable for future manufacturing technologies like additive manufacturing (AM). Cellulose is abundantly available renewable and sustainable raw material. This work focused on studying the properties of thermoplastic cellulose-based composites and their properties using injection molding and 3D printing of granules. The aim was to maximize the cellulose content in composites. Different compounds were prepared using cellulose acetate propionate (CAP) and commercial cellulose acetate propionate with plasticizer $(\mathrm{CP})$ as polymer matrices, microcellulose (mc) and novel cellulose-ester additives; cellulose octanoate (C8) and cellulose palmitate (C16). The performance of compounds was compared to a commercial poly(lactic acid)-based cellulose fiber containing composite. As a result, $\mathrm{CP}$-based compounds had tensile and Charpy impact strength properties comparable to commercial reference, but lower modulus. CP-compounds showed glass transition temperature ( $\mathrm{Tg}$ ) over $58 \%$ and heat distortion temperature (HDT) $12 \%$ higher compared to reference. CAP with $\mathrm{C} 16$ had HDT $82.1^{\circ} \mathrm{C}$. All the compounds were 3D printable using granular printing, but CAP compounds had challenges with printed layer adhesion. This study shows the potential to tailor thermoplastic cellulose-based composite materials, although more research is needed before obtaining all-cellulose 3D printable composite material with high-performance.
\end{abstract}

Keywords: thermoplastic cellulose; cellulose derivative; microcellulose; additive manufacturing; 3D printing; granule printing

\section{Introduction}

Due to the increased concern of environmental issues, novel renewable materials and additive manufacturing (AM) technologies are receiving more and more attention in several industries from medical to transportation industry [1,2]. In addition, the global and European level environmental policies are driving towards more sustainable and recyclable materials $[3,4]$. AM is considered a sustainable and material efficient technology, which uses only the material amount that is needed for the manufacturing of a part or a component, and the amount of waste is therefore low. AM has been used for decades for prototyping and during last years also widely for manufacturing of functional parts [5]. AM enables rapid and cost-effective manufacturing of complex and lightweight parts without any expensive tools such as molds.

AM technologies are divided under seven process categories according to basic principles of each process to create parts layer by layer [6]. The major differences between the process categories are the form of material e.g., powder, liquid, paste, sheets, wire or thermoplastic filaments or granules and the bonding agent or energy which are used in the construction of a part. Material extrusion systems, which are based on melting and dispensing of thermoplastic materials through a nozzle, are widely used in home 3D printing; however, there are also large-scale systems available mainly for industrial 
use [6]. Most of the home 3D printing systems use filaments, however there are many advantages to feed thermoplastic materials in a form of pellets or granules in industrial systems. These include high flow rates and big area prints, very large variety of materials and less expensive material processing costs as filament extrusion and spooling phases can be skipped [7-9].

The environmental issues and technology development have led towards increasing interest to develop novel sustainable high-performance materials for industrial additive manufacturing and for wide variation of applications such as electrical insulation components and parts for automotive and marine industries. Cellulose is a renewable and widely available material and is therefore an attracting alternative especially for fossil-based plastics; however, it is not thermoplastic by nature.

Cellulose is the most abundantly available biopolymer, and it covers up to $50 \mathrm{wt}$ $\%$ of lignocellulosic biomass $[10,11]$. Due to the cellulose inherent properties such as strong hydrogen bonding network, cellulose itself cannot be thermally processed and it is basically insoluble in traditional solvents. In order to impart thermoplastic properties to cellulose, its modification is needed, which is often challenging [12]. Currently well-known cellulose derivatives such as cellulose acetate (CA) and cellulose acetate propionate (CAP) are esterified through cellulose hydroxyl groups. Even though derivatization leads to increased thermoplastic properties their melt-processing window is narrow between melt flow temperature and degradation temperatures, especially with CA [13]. Therefore, quite large quantities of additives, typically plasticizers, are needed to obtain materials suitable for typical thermoplastic processes such as injection molding and extrusion. Long chain cellulose esters are bio-based cellulose esters with a side chain length of C6 or longer [14,15]. It has been reported earlier that for example cellulose octanoate (C8) and cellulose palmitate (C16) are melt processable without any additives [16,17]. These long alkyl side chains have a high plasticizing effect on the cellulose, suggesting that these thermoplastic cellulose compounds can also act as bio-based plasticizers in the composite materials.

The use of composite materials instead of neat polymers generally brings several advantages, such as improved stiffness and high specific strength [18]. Most of the thermoplastic composite materials for extrusion type additive manufacturing use fossil-based polymers such as ABS (acrylonitrile butadiene styrene), but increasing amounts of biobased polymer PLA (polylactic acid) composites have entered to the market [19-22]. PLA is made from renewable raw material, originally from starch. It is an easy-to-print material however it is not very durable and does not stand high temperatures and UV light. The durability and other properties of ABS are clearly better than those of PLA, however ABS is not of renewable origin and releases non-pleasant fumes during printing. The stiffness properties of PLA-based composites are typically enhanced by adding different kind of fillers such as metal powder, carbon or glass-fibers, cellulose, microcellulose, wood and agro-fibers $[23,24]$. However, at the same time, tensile and impact strength properties are decreasing [23,25-28]. Also, due to quite low glass transition temperature, typically below $60{ }^{\circ} \mathrm{C}$, the suitability of PLA-composite in many applications is limited. [29] Lignocellulose materials exhibit many interesting properties, including sustainability, hydrophilicity, biocompatibility, biodegradability, non-toxicity and broad chemical modifying capacity [24]. By using cellulose-based polymers, it is also possible to obtain materials with higher temperature resistance $[30,31]$. In medical applications extrusion printing of thermoplastic cellulose-derivatives for example ethyl cellulose, is a known technology [32,33]. To bring cellulose-based materials also in other applications is an interesting way to proceed.

Several different renewable fillers from continuous flax fibers to nanocellulose have been introduced to composite materials to bring their performance to a desired level. In thermoplastic materials, the typical improvements besides visual outlook and increased renewable material content are in improved material stiffness and specific strength [18]. The main fiber properties affecting composites are related to fiber type, size and aspect ratio. Typically, longer or continuous fibers and fibers with high aspect ratio provide better material properties. However, in material extrusion type manufacturing there are 
limitations to fiber size coming from printing nozzle, which narrows down the selection of suitable fillers. Microcellulose is one material, which due its size and shape is suitable for thermoplastic AM.

In this work, we studied the properties of thermoplastic cellulose-based composites and how they could be applied on novel 3D printing technique, printing of granules. Our aim was to maximize the cellulose content of the composite by using cellulose-based polymer matrix, novel cellulose-based additives as plasticizer and microcellulose to bring improved stiffness to the composite material. To evaluate the property level compared to existing commercial materials we used a commercial PLA-based cellulose containing composite material as a reference.

\section{Results and Discussion}

\subsection{Test Series}

Test series for additive manufacturing contained seven different compounds and two reference materials presented in Table 1 . The following acronyms have been used in the coding: microcellulose $(\mathrm{mc})$, commercial thermoplastic cellulose acetate prionate polymer with $17 \%$ commercial plasticizer (CP), thermoplastic cellulose esters; cellulose octanoate (C8) and cellulose palmitate (C16), cellulose acetate propionate without added plasticizer (CAP) and commercial printable PLA-based cellulose composite material reference (Cref).

Table 1. Test series of compounds.

\begin{tabular}{|c|c|c|c|c|}
\hline Code & $\begin{array}{c}\text { Thermoplastic Cellulose } \\
\text { Ester (C) Type }\end{array}$ & $\begin{array}{l}\text { Thermoplastic Cellulose } \\
\text { Ester (C) Content, (\%) }\end{array}$ & $\begin{array}{l}\text { Cellulose Fibre } \\
\text { Content, }(\%)\end{array}$ & Description \\
\hline $\mathrm{CP}$ & & 0 & 0 & $\begin{array}{l}\text { Commercial } \\
\text { thermoplastic cellulose } \\
\text { polymer }\end{array}$ \\
\hline CP-mc & & 0 & 20 & \\
\hline $\mathrm{CP}-\mathrm{mc}-\mathrm{C} 8$ & $\mathrm{C} 8$ & 4 & 20 & \\
\hline CP-mc-C16 & $\mathrm{C} 16$ & 4 & 20 & \\
\hline CAP-C8 & $\mathrm{C} 8$ & 17 & 0 & $\begin{array}{c}\text { CAP without } \\
\text { commercial plasticizer }\end{array}$ \\
\hline CAP-C16 & $\mathrm{C} 16$ & 17 & 0 & $\begin{array}{c}\text { CAP without } \\
\text { commercial plastizicer }\end{array}$ \\
\hline CAP-mc-C8 & $\mathrm{C} 8$ & 17 & 20 & $\begin{array}{c}\text { CAP without } \\
\text { commercial plastizicer }\end{array}$ \\
\hline CAP-mc-C16 & C16 & 17 & 20 & $\begin{array}{c}\text { CAP without } \\
\text { commercial plastizicer }\end{array}$ \\
\hline Cref & & 0 & 20 & $\begin{array}{l}\text { Commercial cellulose } \\
\text { fibre containing } \\
\text { reference material }\end{array}$ \\
\hline
\end{tabular}

All materials in Table 1 were injection molded to see the ultimate properties of the compounds without any effect originating from the $3 \mathrm{D}$ printing process. The $3 \mathrm{D}$ printing of materials was performed using granule printing (GP) process.

\subsection{Results from the Printing Tests}

Print head of the 3D printing system and granules used in the 3D printing are shown in the Figure 1.3D printability of the material compositions was tested, while simultaneously manufacturing the specimens for tensile and impact strength tests. Descriptions of 3D printability of all the material compositions and images showing the 3D printing results and challenges are collected in Table 2. 3D printability described with "good" means that 
there was a constant material flow and good enough adhesion between the layers so that it was possible to manufacture $3 \mathrm{D}$ objects.
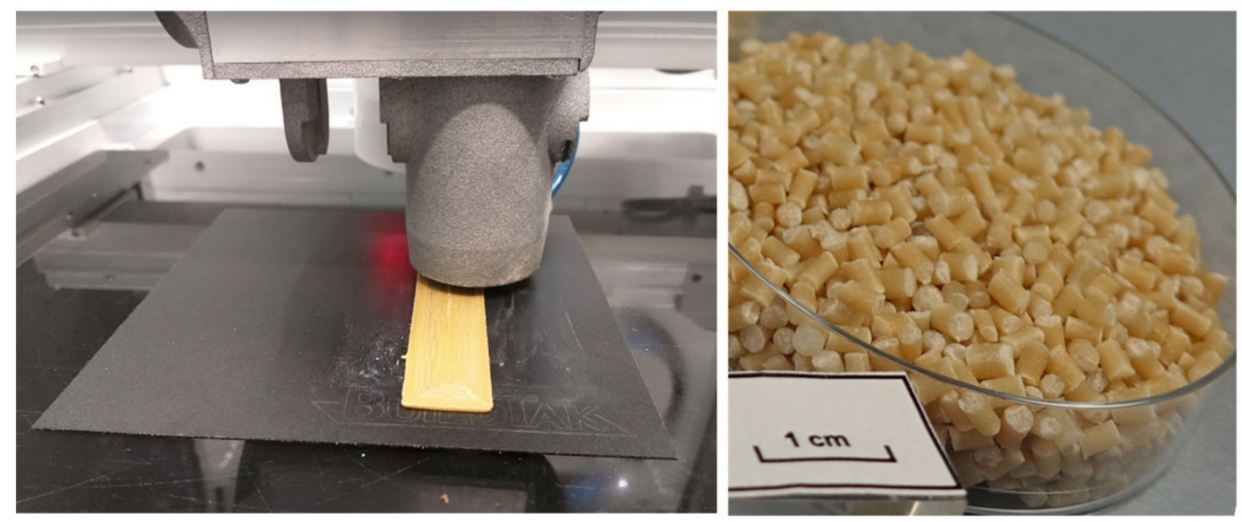

Figure 1. Granulate 3D printing head (left) and granules used in 3D printing process (right).

Table 2. Results from 3D printing trials with granule printing method.

\begin{tabular}{ccc}
\hline Code & 3D printability & Image \\
\hline $\mathrm{CP}$ & Good & \\
\hline $\mathrm{CP}-\mathrm{mc}$ & Good & \\
\hline $\mathrm{CP}-\mathrm{mc}-\mathrm{C} 8$ & Good & \\
\hline $\mathrm{CP}-\mathrm{mc}-\mathrm{C} 16$ & Good & \\
\hline
\end{tabular}

CAP-C8 Good, however poor layer adhesion, oily material, fragile

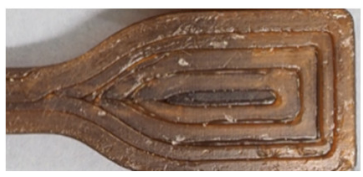

CAP-C16 Limited, however poor layer adhesion, oily material, fragile

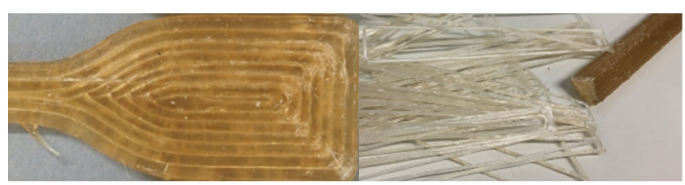

CAP-mc-C8 Limited, and poor layer adhesion, oily material

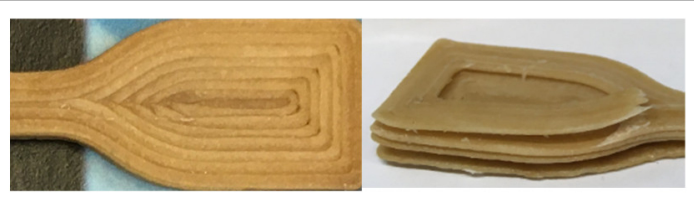


Table 2. Cont.

\begin{tabular}{ccc}
\hline Code & 3D printability & Image \\
\hline CAP-mc-C16 & Good, however poor layer adhesion, oily material, \\
fragile & \\
\hline Cref & Good \\
\hline
\end{tabular}

The results from the 3D printing trials with granule printing are showing good printability for reference commercial thermoplastic cellulose (CP), PLA-cellulose composite (Cref) and materials having commercial cellulose polymer with plasticizer $(\mathrm{CP})$ as the polymer base. Most of the materials based on cellulose polymer without plasticizer (CAP) were $3 \mathrm{D}$ printable, however the 3D printed samples were very fragile. The fragile structure was a result of a poor adhesion between the 3D printed strings and layers. These materials with limited layer adhesion had an oily surface explored with fingers contrary to $\mathrm{CP}$-materials with a drier and fibrous character. The most limited 3D printability was observed with CAP-mc-C 8 and CAP-C16 of which there are extra images in the Table 2 showing the poor layer adhesion.

It is highlighted that in this study the main object was to compare different material compositions and to manufacture samples using 3D printing for further analysis and testing. For that reason, most of the 3D printing parameters were kept unchanged after a careful pre-testing of those. We believe that the properties of the 3D printed samples can be further enhanced by exploring and optimisation of the different 3D printing strategies and parameters in the future. Especially the quality of the 3D printing can be fine-tuned with cellulose composite materials having a good 3D printability.

\subsection{Visual Results for Injection Molded Samples}

All materials were injection molded to standard dog-bone shaped tests bars presented in Figure 2, that shows the visual difference between materials and some challenges caused by thermal stress.

The injection molded materials in Figure 2 are showing more clearly the inherent properties of the materials, for example color. It was relatively easy to produce the test specimens from all of the materials using injection molding. CP, CAP-C 8 and CAP-C16 were all transparent. The dark color of the materials containing modified cellulose additive, C8, is partly coming from the brownish color of the additive itself. However, the two thermal stress cycles during compounding and injection molding at max $205{ }^{\circ} \mathrm{C}$ seem to cause some color formation, but probably not real degradation as their degradation temperature, presented in Table 3, is much higher than the processing temperatures. Also, the degradation of C8, explained in Section 2.5.1. occurs at higher temperature than used in injection molding. The cellulose-ester additive used in amounts of $4 \%$ in the mc containing $\mathrm{CP}$ compounds (CP-mc-C8, CP-mc-C16) lead to improved mc dispersion compared to CAP-mc-C8 and CAP-mc-C16 with $17 \%$ cellulose-ester additives. When comparing $\mathrm{CP}-\mathrm{mc}$ and $\mathrm{CP}-\mathrm{mc}-\mathrm{C} 16$ the latter one had less color formation, probably due to higher plasticizer/additive content, $13 \%$ vs. $17 \%$. This suggests that small addition of cellulose-ester, especially $\mathrm{C} 16$, has beneficial effect on mc dispersion and color formation in compound. Lightest color was in PLA-based Cref. 


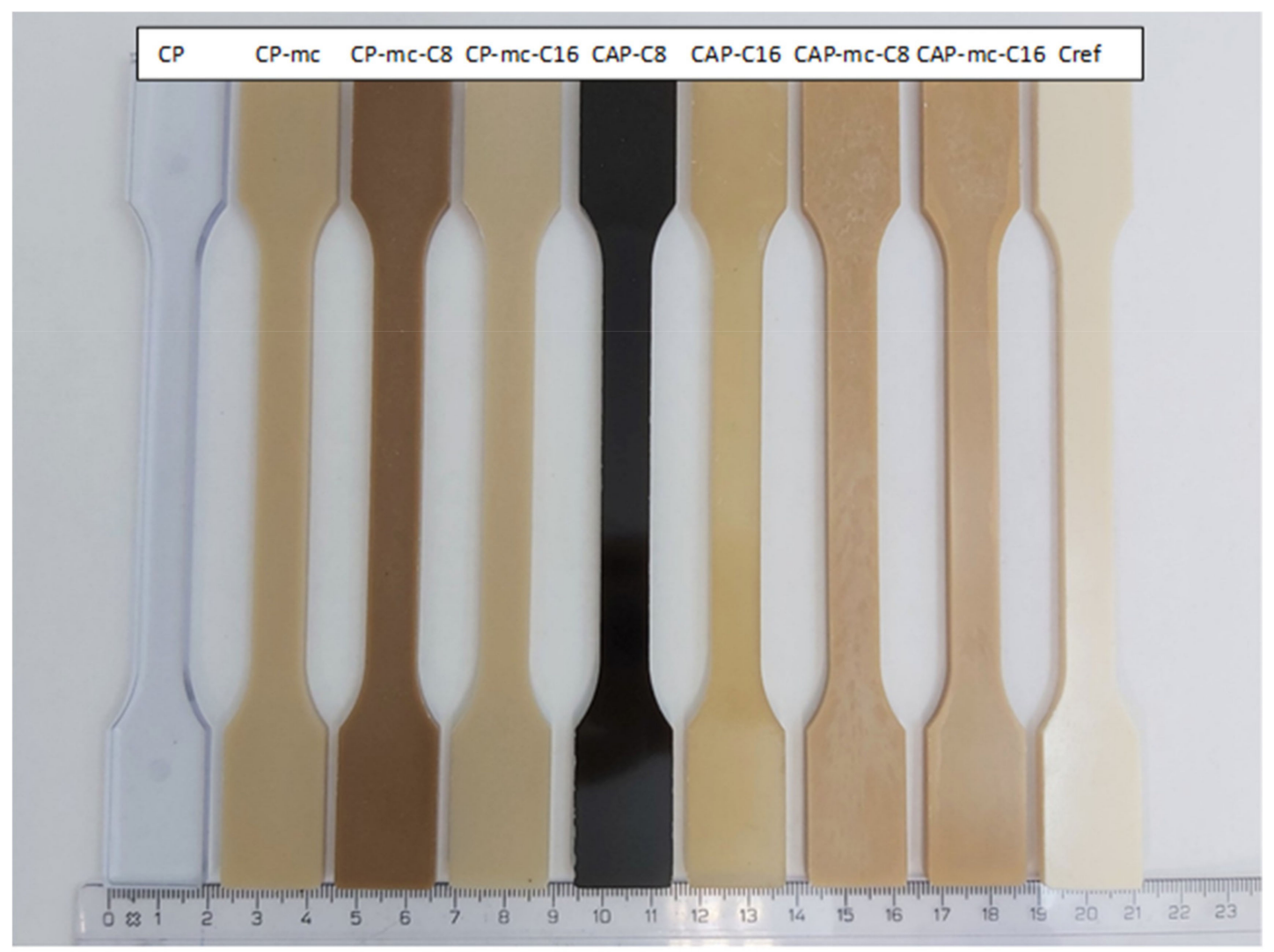

Figure 2. Injection molded test bars.

Table 3. Results from DSC and TGA analytics. DSC-results are from the first heating, cooling and second heating cycles for the compounded granules. TGA results are for the temperature, where $5 \%$ of the material is degraded.

\begin{tabular}{|c|c|c|c|c|c|c|}
\hline \multirow[t]{2}{*}{ Code } & \multicolumn{2}{|c|}{ First Heating } & \multirow{2}{*}{$\begin{array}{c}\text { Cooling } \\
\mathrm{Tc}^{\circ}{ }^{\circ} \mathrm{C}\end{array}$} & \multicolumn{2}{|c|}{ Second Heating } & \multirow{2}{*}{$\begin{array}{c}\text { TGA, 5\% Degraded } \\
\text { Tdeg },{ }^{\circ} \mathrm{C}\end{array}$} \\
\hline & Tsoft, ${ }^{\circ} \mathrm{C}$ & $\mathrm{Tm},{ }^{\circ} \mathrm{C}$ & & $\mathrm{Tg},{ }^{\circ} \mathrm{C}$ & $\mathrm{Tm},{ }^{\circ} \mathrm{C}$ & \\
\hline $\mathrm{CP}$ & 42 & 158 & 122 & 95 & 159 & 267 \\
\hline $\mathrm{CP}-\mathrm{mc}$ & 47 & 157 & 124 & 97 & 160 & 273 \\
\hline $\mathrm{CP}-\mathrm{mc}-\mathrm{C} 8$ & 40 & 161 & 123 & 103 & 160 & 264 \\
\hline CP-mc-C16 & 34 & $114 / 161$ & n.d. & 103 & 163 & 277 \\
\hline CAP-C8 & 54 & $173 / 191$ & n.d. & 55 & $154 / 181$ & 267 \\
\hline CAP-C16 & 36 & $173 / 206$ & n.d. & 32 & $146 / 172$ & 298 \\
\hline CAP-mc-C8 & 72 & $161 / 182$ & 145 & 78 & $142 / 194$ & 309 \\
\hline CAP-mc-C16 & 34 & $98 / 168$ & n.d. & 84 & $175 / 194$ & 305 \\
\hline Cref & 61 & 149 & 125 & 60 & 165 & 304 \\
\hline
\end{tabular}

Standard deviation in DSC-method: $\pm 2{ }^{\circ} \mathrm{C}$ (DIN 53765).

\subsection{Scanning Electron Microscopy (SEM)}

SEM-images of injection molded samples with $100 \times$ and $2500 \times$ enlargements are presented in Figures 3 and 4, and 3D printed samples with $100 \times$ enlargement in Figure 5. The images are taken from the cross-cut surface in the middle of the test bar to see the dispersion of the fibers inside the material and to avoid the surface smoothing effect originating from the manufacturing process. 

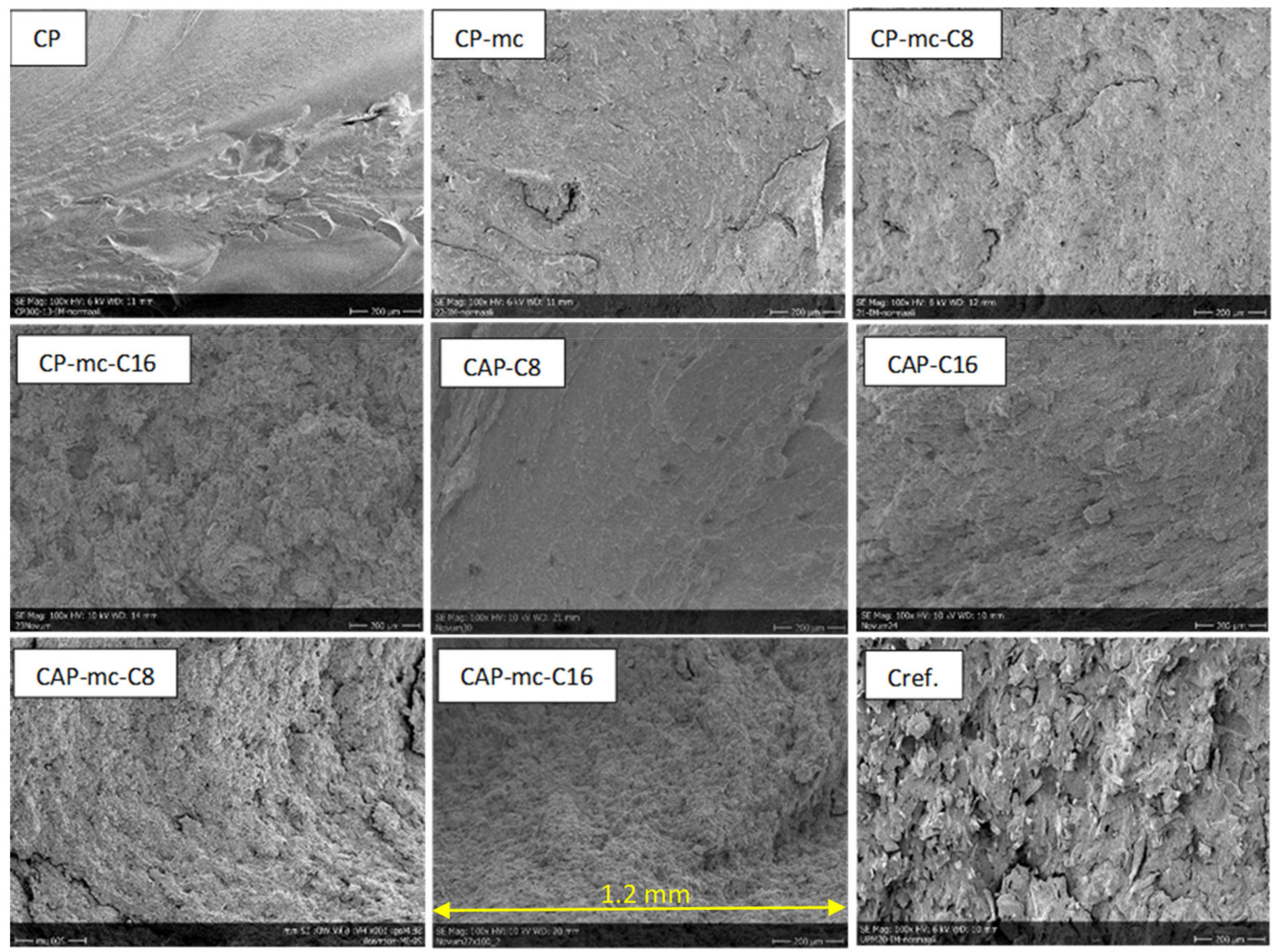

Figure 3. SEM-pictures with $100 \times$ enlargement from cross-cut middle of the injection molded test bars. The width of each pictures is $1.2 \mathrm{~mm}$. CP is commercial cellulose acetate propionate with $13 \%$ commercial plasticizer; CP-mc is $20 \%$ microcellulose containing CP; CP-mc-C8 contains also $4 \%$ cellulose octanoate C8; CP-mc-C16 contains $4 \%$ of cellulose palmitate $\mathrm{C} 16$; CAP-C 8 contains neat cellulose acetate propionate (CAP) with 17\% C8; CAP-C16 contain $17 \%$ C16 in CAP; CAP-mc-C 8 contains 20\% mc and 17\% C8 in CAP; CAP-mc-C16 contains 20\% mc and 17\% C16 in CAP and Cref is PLA-based material with $20 \%$ cellulose fibre.

The SEM-images of the cross-cut of injection moulded materials with $100 \times$ enlargement in Figure 3 are showing the quite homogeneous structure of mc containing materials compared to Cref, where the the fibres are sticking out from the polymer structure.

SEM-images from the cross-cut of injection molded materials with $2500 \times$ enlargement in Figure 4 are showing how C8 and C16 addition, in amounts of $17 \%$, on CAP makes the material flaky, if we compare it with $\mathrm{CP}$, containing $13 \%$ of commercial additive. This might be due to some miscibility challenges of $\mathrm{C} 8$ and $\mathrm{C} 16$ with CAP. The longer the carbon chain in the cellulose additive is, the more flaky the compound with CAP is. This can be seen also in CAP-C8 and CAP-C16 images in Table 2, where it is described oiliness of these two materials and challenges of CAP-C16 layers to stick to each other. In mc containing materials with $\mathrm{C} 8$ and $\mathrm{C} 16$ (CP-mc-C8, CP-mc-C16, CAP-mc-C8 and CAP-mc-C16) the SEM-images of materials resemble each other and $\mathrm{mc}$ is quite tightly connected to cellulose polymer. Instead, in CP-mc, without the cellulose-based additive, $\mathrm{mc}$ is clearly visible as well as cellulose fibers in Cref, and both have visible gaps between fiber and polymer. The connection between the fiber and the polymer seems to be tighter with $\mathrm{CP}$ in CP-mc than with PLA in Cref. 

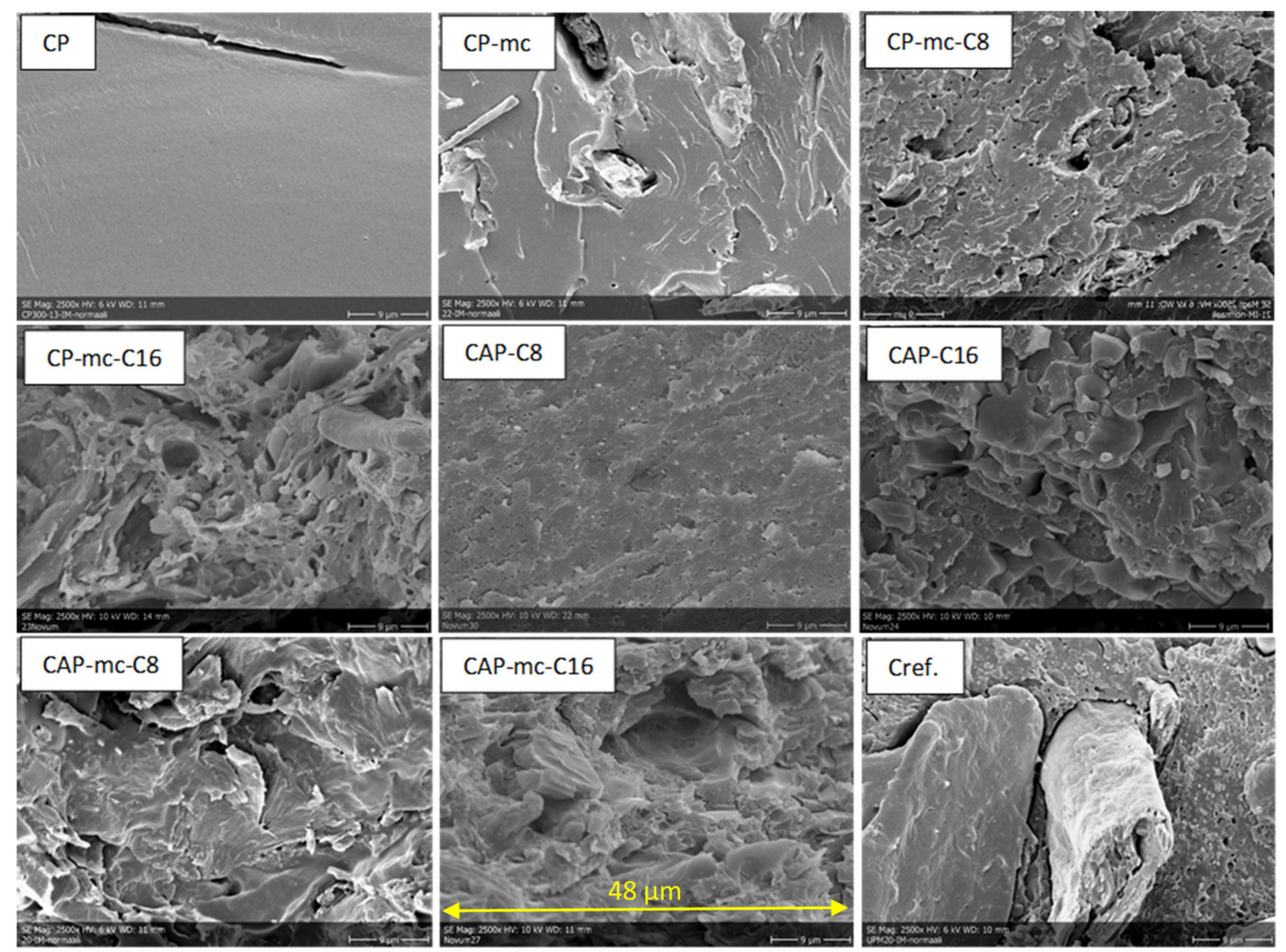

Figure 4. SEM-pictures with $2500 \times$ enlargement from cross-cut middle of the injection molded test bars. The width of each pictures is $48 \mu \mathrm{m}$. CP is commercial cellulose acetate propionate with $13 \%$ commercial plasticizer; CP-mc is $20 \%$ microcellulose containing CP; CP-mc-C8 contains also $4 \%$ cellulose octanoate C8; CP-mc-C16 contains $4 \%$ of cellulose palmitate $\mathrm{C} 16$; $\mathrm{CAP}-\mathrm{C} 8$ contains neat cellulose acetate propionate (CAP) with 17\% C8; CAP-C16 contain $17 \% \mathrm{C} 16$ in CAP; CAP-mc-C 8 contains $20 \%$ mc and 17\% C8 in CAP; CAP-mc-C16 contains 20\% mc and 17\% C16 in CAP and Cref is PLA-based material with $20 \%$ cellulose fibre.

SEM-images with $100 \times$ enlargement from the cross-cut of 3D-printed test bars in Figure 5 show the attachment between printed layers, but also significant amount of porosity inside the materials especially where fibers are included. The neat commercial polymer $\mathrm{CP}$ has good attachment between layers, but still some gaps in between the separate printed lines. CAP-C8 shows the same flaky cross-cut surface as with injection molded material. In the case of CAP-C16, the picture was taken from the side of the test bar due to total breakdown during sample preparation. Even this picture from the side of the CAP-C16 shows separation or poor attachment of the printed layers originating from the oily surface of the material containing $17 \%$ of cellulose-ester additive C16. However, C16 containing materials with mc (CP-mc-C16 and CAP-mc-C16) had both good printability as mentioned in Table 2. It is possible that mc absorbs part of the oily $\mathrm{C} 16$ and even improves mc dispersion inside the compound. In CP-mc-C16 the C16 amount was 4\%, and thus significantly lower than $17 \%$ in the CAP-mc-C16, but probably still high enough to provide improved plasticization and fiber dispersion if compared to CP-mc without additional cellulose-ester additive. The layer adhesion, in general, was better in commercial polymer, $\mathrm{CP}$-based compounds with $13 \%$ commercial plasticizer, as compared to CAP, where the only plasticizer was cellulose-ester additive in amounts of $17 \%$. The layer adhesion was also good with commercial PLA-based compound Cref. The challenges in interlayer bond formation in 3D printing especially focused on filament printing, is discussed more widely for example by Lamm et al. [34]. However, similar regularities are relevant also in granular 
printing and maybe even in bigger scale, because there is one process step less, filament manufacturing.
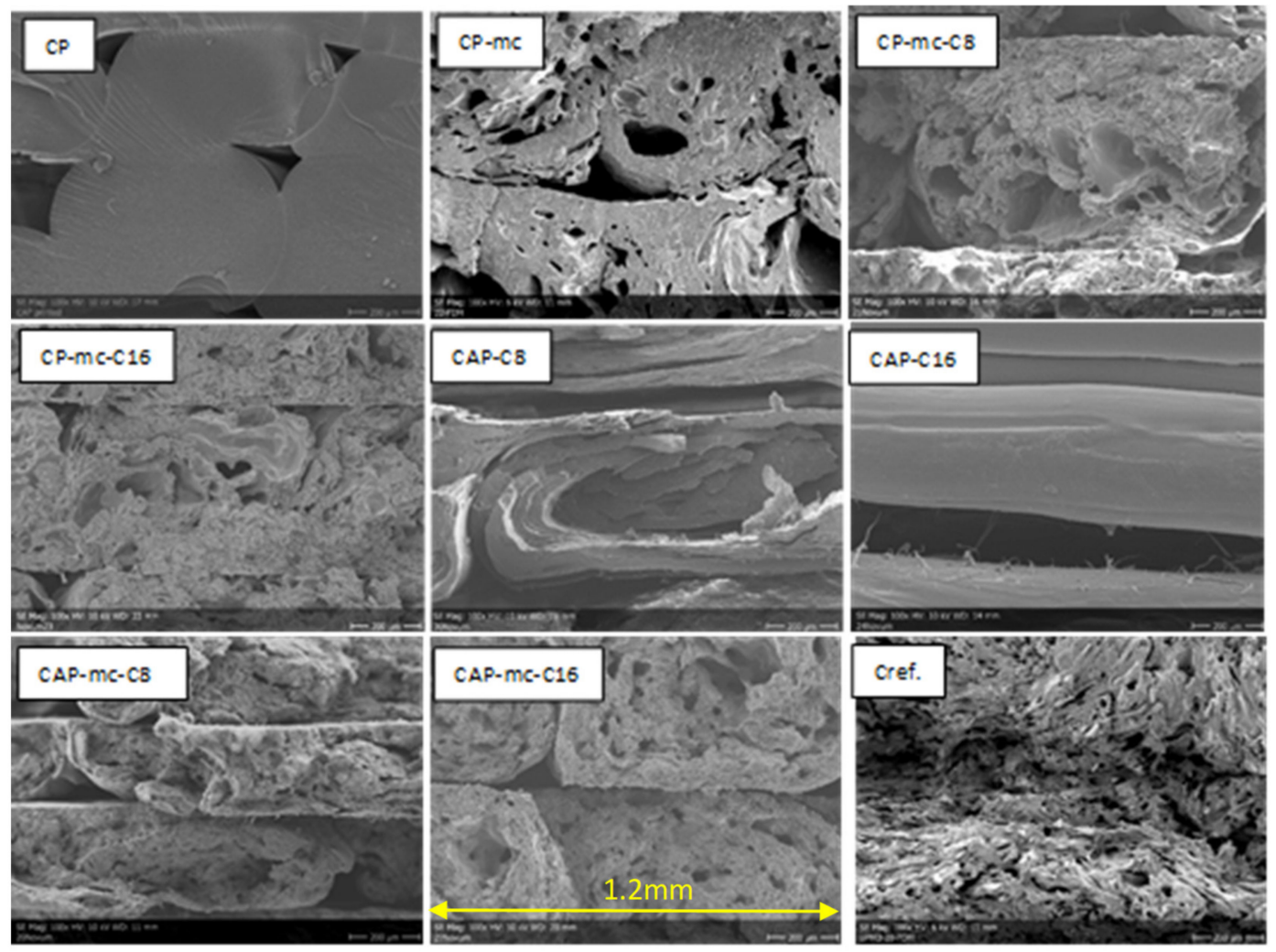

Figure 5. SEM-pictures with $100 \times$ enlargement from the cross-cut of 3D printed test bars. CAP-C16 is from the side of the printed test bar. The width of each pictures is $1.2 \mathrm{~mm}$. CP is commercial cellulose acetate propionate with $13 \%$ commercial plasticizer; CP-mc is $20 \%$ microcellulose containing CP; CP-mc-C8 contains also $4 \%$ cellulose octanoate C8; CP-mc-C16 contains $4 \%$ of cellulose palmitate C16; CAP-C 8 contains neat cellulose acetate propionate (CAP) with 17\% C8; CAP-C16 contain 17\% C16 in CAP; CAP-mc-C8 contains 20\% mc and 17\% C8 in CAP; CAP-mc-C16 contains $20 \% \mathrm{mc}$ and $17 \% \mathrm{C} 16$ in CAP and Cref is PLA-based material with $20 \%$ cellulose fibre.

\subsection{Thermal Resistance Related Results}

2.5.1. Differential Scanning Calorimetry (DSC) and Thermogravimetric Analysis (TGA) Results

DSC analytics was made to see how the addition of mc, C8 and C16 affect the softening of the material (Tsoft), glass transition (Tg), melting (Tm) and crystallization (Tc) behavior of the composite materials. The material processing to products is going through two heating cycles, when granule printing is used (compounding and 3D-printing). By using DSC it is possible to see, if the material stabilizes within these heating cycles. TGA analytics was made to see, if the materials were stable in the processing temperatures and no degradation occurred. The DSC results for two heating runs and one cooling run are presented in Table 3 together with the TGA-results for the temperature where $5 \%$ of the material is degraded.

The results from the DSC-analysis indicate that some softening of the cellulose polymer-based materials starts already in temperatures above $34{ }^{\circ} \mathrm{C}$ during the first heating of the granule. This is connected to the mobility of the plasticizer in CP-based materials and softening of the plasticizing cellulose-ester additive side chains, C8 and C16, in CAP-based materials. The two melting peaks in CAP-based materials are related to additive melting and CAP-polymer melting (188-210 ${ }^{\circ} \mathrm{C}$ according to producer information). Typically, long chain thermoplastic cellulose-esters are amorphous materials, so their melting point is not clear. However, side chain crystallization and melting can occur when the length of the 
side chain is $\mathrm{C} 10$ or greater [35]. For cellulose palmitate (C16), side chain melting has been reported to be $20-35^{\circ} \mathrm{C}$ depending on DS. $[14,35,36]$ The two melting peaks in CAP based materials indicate poor miscibility of the thermoplastic cellulose-esters with CAP, that could be also seen in the SEM-image, Figure 4, for CAP-C8 and CAP-C16. The addition of cellulose-ester additive C 8 and especially $\mathrm{C} 16$ is increasing amorphous character of compounds, which can be detected through smaller or disappearing crystalline changes during cooling of the material containing $\mathrm{C} 8$ or $\mathrm{C} 16$.

During the second heating the softening of $\mathrm{CP}$-based materials in low temperatures seems to disappear and there are $\mathrm{Tg}^{\prime} \mathrm{s}$ close to $100^{\circ} \mathrm{C}$, that can indicate the stabilization of compounds during increasing heating cycles. Moreover, the one melting point indicates better misciblity of cellulose-based additives $\mathrm{C} 8$ and $\mathrm{C} 16$ with $\mathrm{CP}$ than with neat CAP. Compared to Cref, with $\mathrm{Tg}$ about $60^{\circ} \mathrm{C}$ in both heating cycles, the cellulose-based compounds with mc seem to endure higher temperatures after second heating, which corresponds to readymade product after injection molding or extrusion manufacturing process.

TGA was analyzed to see if the materials can tolerate manufacturing temperatures up to $225^{\circ} \mathrm{C}$ without significant degradation. According to results presented in Table 3 it is possible to use higher processing temperatures than normally suggested to cellulose fibers containing materials, below $200{ }^{\circ} \mathrm{C}$. [18] Degradation temperatures of pure cellulose-based additives $\mathrm{C} 8$ and $\mathrm{C} 16$ have been reported to be between $230-270{ }^{\circ} \mathrm{C}$ [16] and are therefore clearly higher than the processing temperatures used in this research.

\subsubsection{Heat Distortion Temperature (HDT) Analysis}

Heat distortion temperature (HDT) was analyzed for the injection molded materials to avoid the possible error coming from poor layer adhesion and to see the inherent properties coming only from the material. The results are presented in Table 4 and Figure 6.

Table 4. HDT-results for injection molded materials.

\begin{tabular}{cc}
\hline Code & HDT, ${ }^{\circ} \mathbf{C}$ \\
\hline CP & $58 \pm 0.8$ \\
\hline CP-mc & $58.6 \pm 0.3$ \\
\hline CP-mc-C8 & $59.6 \pm 0.3$ \\
\hline CP-mc-C16 & $62.1 \pm 0.9$ \\
\hline CAP-C8 & $68.6 \pm 5.6$ \\
\hline CAP-C16 & $82.1 \pm 0.7$ \\
\hline CAP-mc-C8 & $74.8 \pm 2.3$ \\
\hline CAP-mc-C16 & $72.9 \pm 2.4$ \\
\hline Cref & $55.4 \pm 0.8$ \\
\hline
\end{tabular}

Heat distortion temperature (HDT) is one of most important properties of polymeric materials indicating at what temperature the material starts to soften under a specific load. When comparing the HDT results in Table 4 and Figure 6 we can see that the commercial reference compound PLA-based Cref, has HDT value $55.4^{\circ} \mathrm{C}$ and the commercial cellulose polymer $\mathrm{CP}\left(58^{\circ} \mathrm{C}\right)$ and $\mathrm{CP}-\mathrm{mc}\left(58.6^{\circ} \mathrm{C}\right)$ are in the same level. For many applications those are quite low values. By adding cellulose-ester additive $\mathrm{C} 16$, the HDT was improved by $7 \%$ to $62.1^{\circ} \mathrm{C}$ and it looks that C16 provides higher values compared to C8 cellulose-ester additive. When $\mathrm{C} 16$ is used as the only plasticizer/additive for CAP in CAP-C16, the HDT value was increased by $42 \%$ to $82.1^{\circ} \mathrm{C}$ compared to CP. In mc containing compounds CAP-mc-C8 and CAP-mc-C16 the increase was $35 \%$ to $32 \%$ compared to Cref, respectively. By modifying CAP-based compound with different plasticizers, it seems possible to obtain materials with improved temperature resistance and that way to widen the applicability potential of thermoplastic cellulose composites. 


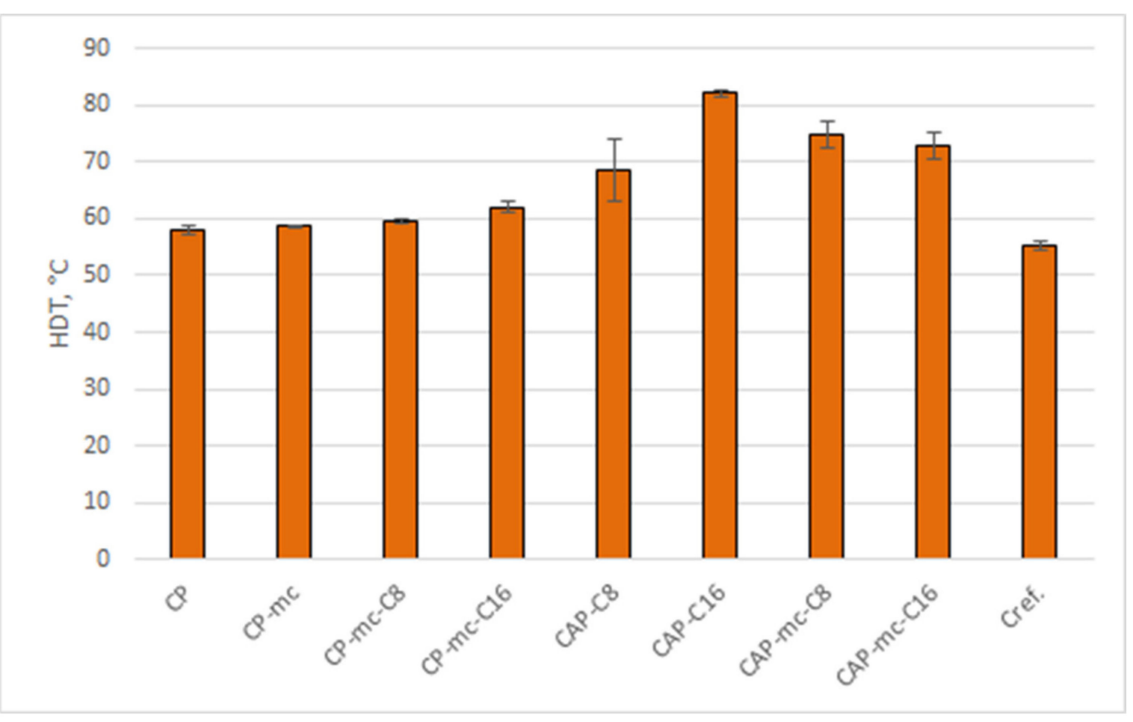

Figure 6. Heat distortion temperatures for injection molded materials. $\mathrm{CP}$ is commercial cellulose acetate propionate with $13 \%$ commercial plasticizer, $\mathrm{CP}-\mathrm{mc}$ is $20 \%$ microcellulose containing $\mathrm{CP}, \mathrm{CP}-\mathrm{mc}-\mathrm{C} 8$ contains also $4 \%$ cellulose octanoate $\mathrm{C} 8$, CP-mc-C16 contains $4 \%$ of cellulose palmitate C16, CAP-C8 contains neat cellulose acetate propionate (CAP) with $17 \% \mathrm{C} 8$, CAP-C16 contain 17\% C16 in CAP, CAP-mc-C8 contains $20 \% \mathrm{mc}$ and $17 \%$ C8 in CAP, CAP-mc-C16 contains $20 \%$ mc and $17 \%$ C16 in CAP and Cref is PLA-based material with $20 \%$ cellulose fibre.

\subsection{Results from the Mechanical Tests}

The mechanical strength tests such as tensile strength and Charpy impact strength values were analyzed for both injection molded materials and 3D granule printed test samples. The results for injection molded materials are showing the ultimate strength properties for each material. Results for 3D granule printed materials are reflecting also attachments of printed layers and internal porosity as well as fiber-polymer attachment of the material.

\subsubsection{Tensile Strength}

Results from the tensile strength tests for injection molded and 3D printed materials are presented in Table 5. The separate results for tensile strength at yield are visualized in Figure 7, results for Young's modulus in Figure 8 and results for strain at yield in Figure 9.

Table 5. Results from tensile strength test for injection molded and 3D printed materials.

\begin{tabular}{|c|c|c|c|c|c|c|}
\hline & \multicolumn{3}{|c|}{ Injection Moulded } & \multicolumn{3}{|c|}{ Granule Printed } \\
\hline & $\begin{array}{c}\text { Strength at Yield, } \\
\mathrm{MPa}\end{array}$ & $\begin{array}{c}\text { Young's } \\
\text { Modulus, MPa }\end{array}$ & Strain at Yield, \% & $\begin{array}{c}\text { Strength at Yield, } \\
\mathrm{MPa}\end{array}$ & $\begin{array}{c}\text { Young's } \\
\text { Modulus, MPa }\end{array}$ & Strain at Yield, \% \\
\hline $\mathrm{CP}$ & $26.0 \pm 0.3$ & $1360 \pm 25$ & $3.5 \pm 0.04$ & $23.6 \pm 2.5$ & $1297 \pm 121$ & $3.9 \pm 0.2$ \\
\hline $\mathrm{CP}-\mathrm{mc}$ & $28.9 \pm 0.1$ & $1710 \pm 45$ & $3.0 \pm 0.04$ & $11.6 \pm 0.7$ & $902 \pm 42$ & $2.5 \pm 0.2$ \\
\hline CP-mc-C8 & $26.0 \pm 0.1$ & $1670 \pm 74$ & $2.9 \pm 0.1$ & $8.8 \pm 1.3$ & $837 \pm 54$ & $0.9 \pm 0.7$ \\
\hline CP-mc-C16 & $25.5 \pm 0.1$ & $1840 \pm 69$ & $2.8 \pm 0.04$ & $12.9 \pm 1.3$ & $977 \pm 81$ & $2.5 \pm 0.3$ \\
\hline CAP-C8 & $10.9 \pm 0.4$ & $1539 \pm 65$ & $0.8 \pm 0.04$ & $6.7 \pm 1.0$ & $1297 \pm 307$ & $0.3 \pm 0.2$ \\
\hline CAP-C16 & $29.0 \pm 2.2$ & $1680 \pm 87$ & $2.2 \pm 0.2$ & $10.0 \pm 1.9$ & $1115 \pm 185$ & $0.3 \pm 0.7$ \\
\hline CAP-mc-C 8 & $31.8 \pm 1.0$ & $2034 \pm 116$ & $2.1 \pm 0.2$ & $11.0 \pm 2.1$ & $2147 \pm 717$ & $0.3 \pm 0.4$ \\
\hline CAP-mc-C16 & $24.0 \pm 2.8$ & $1981 \pm 51$ & $1.5 \pm 0.3$ & $5.4 \pm 1.1$ & $1690 \pm 274$ & $0.4 \pm 0.1$ \\
\hline Cref & $26.6 \pm 0.2$ & $2642 \pm 329$ & $2.1 \pm 0.2$ & $11.6 \pm 0.3$ & $1754 \pm 133$ & $1.6 \pm 0.3$ \\
\hline
\end{tabular}




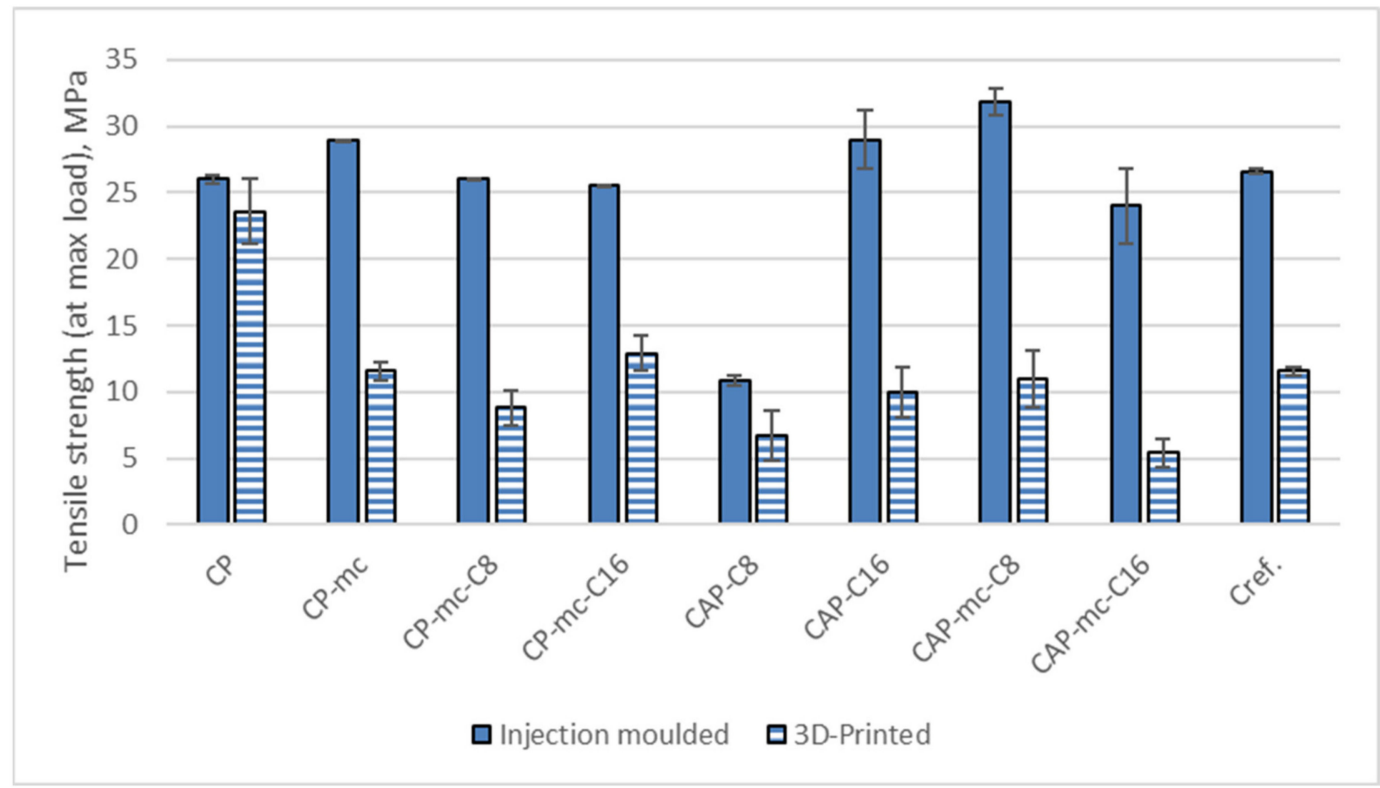

Figure 7. Tensile strength at yield for injection molded and 3D printed materials. $\mathrm{CP}$ is commercial cellulose acetate propionate with $13 \%$ commercial plasticizer; CP-mc is $20 \%$ microcellulose containing CP; CP-mc-C 8 contains also $4 \%$ cellulose octanoate $\mathrm{C} 8$; CP-mc-C16 contains $4 \%$ of cellulose palmitate $\mathrm{C} 16$; CAP-C8 contains neat cellulose acetate propionate (CAP) with 17\% C8; CAP-C16 contain 17\% C16 in CAP; CAP-mc-C8 contains $20 \%$ mc and 17\% C8 in CAP; CAP-mc-C16 contains $20 \% \mathrm{mc}$ and $17 \% \mathrm{C} 16$ in CAP and Cref is PLA-based material with $20 \%$ cellulose fibre.

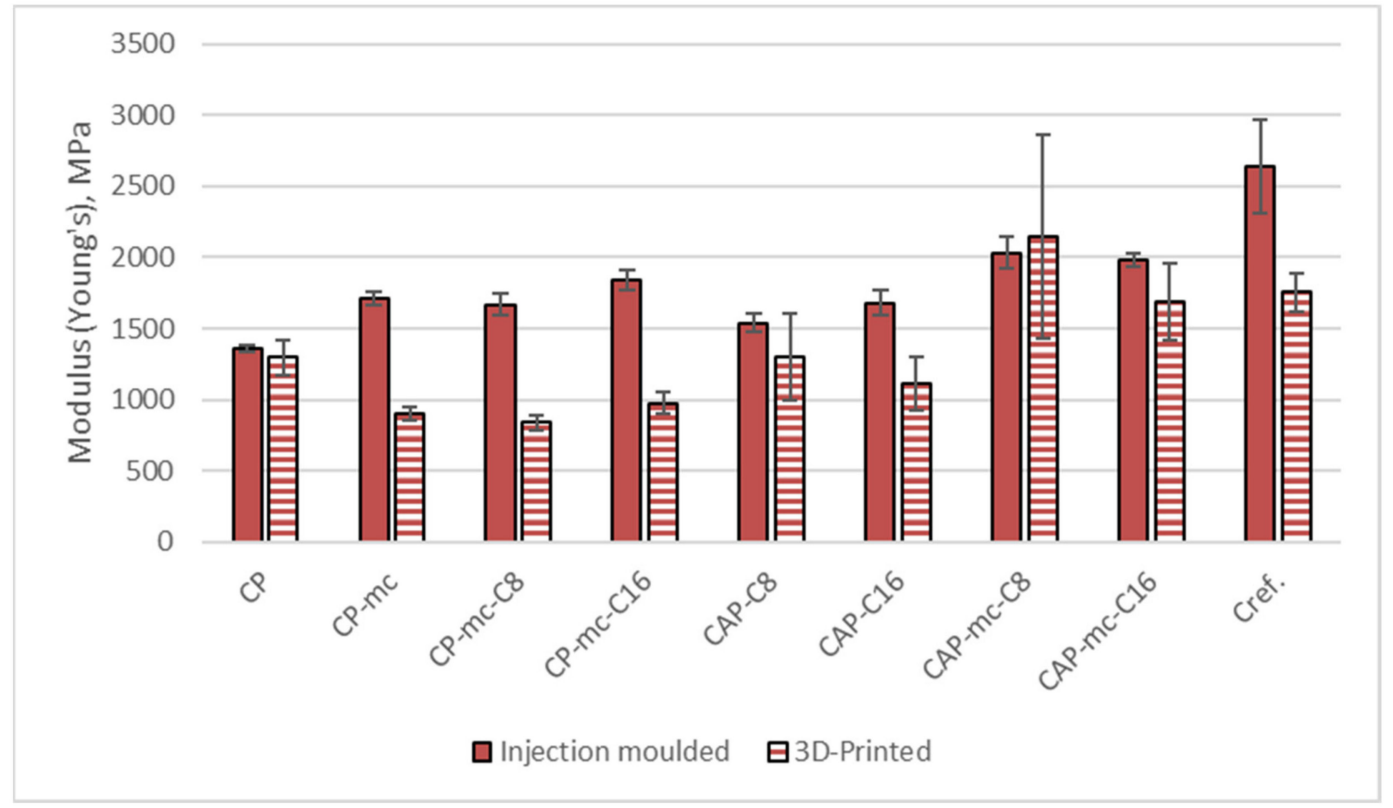

Figure 8. Young's modulus for injection molded and 3D printed materials. $\mathrm{CP}$ is commercial cellulose acetate propionate with $13 \%$ commercial plasticizer; CP-mc is $20 \%$ microcellulose containing CP; CP-mc-C 8 contains also $4 \%$ cellulose octanoate C8; CP-mc-C16 contains $4 \%$ of cellulose palmitate C16; CAP-C8 contains neat cellulose acetate propionate (CAP) with $17 \%$ C8; CAP-C16 contain 17\% C16 in CAP; CAP-mc-C8 contains 20\% mc and 17\% C8 in CAP; CAP-mc-C16 contains $20 \%$ mc and $17 \%$ C16 in CAP and Cref is PLA-based material with $20 \%$ cellulose fibre. 


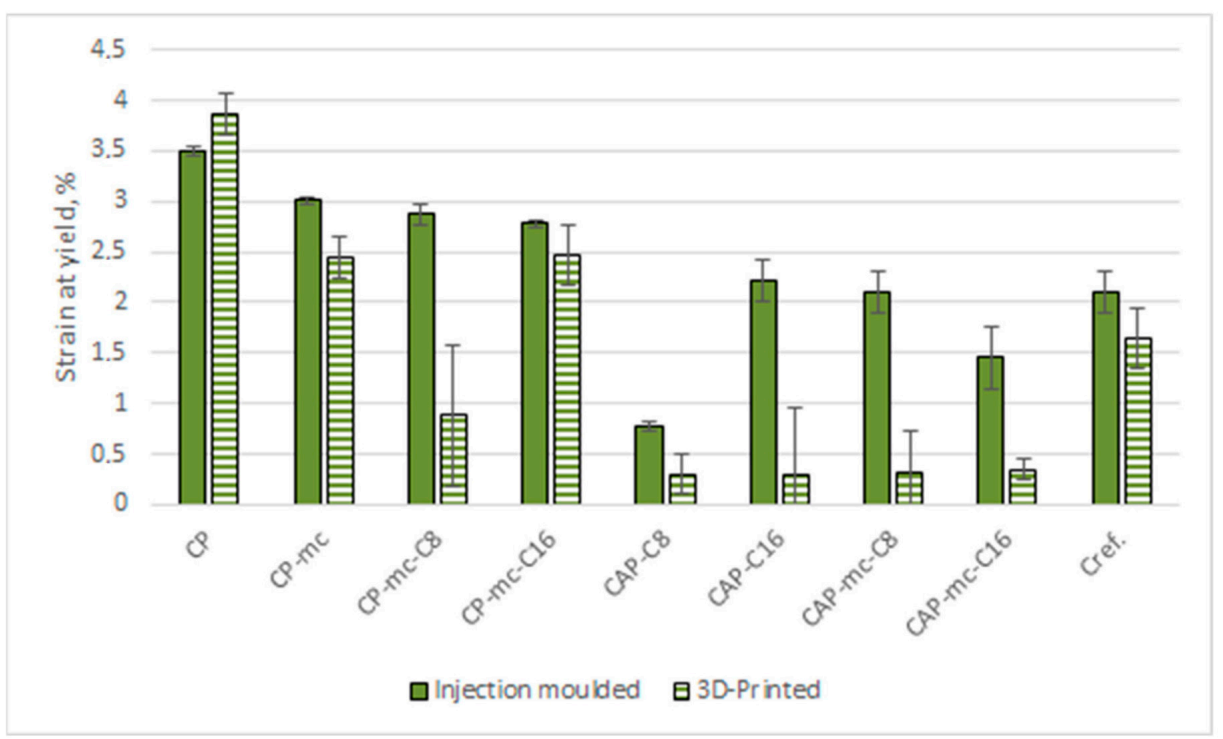

Figure 9. Strain at yield for injection molded and 3D printed materials. $\mathrm{CP}$ is commercial cellulose acetate propionate with $13 \%$ commercial plasticizer; CP-mc is $20 \%$ microcellulose containing CP; CP-mc-C8 contains also $4 \%$ cellulose octanoate C8; CP-mc-C16 contains $4 \%$ of cellulose palmitate C16; CAP-C8 contains neat cellulose acetate propionate (CAP) with $17 \%$ C8; CAP-C16 contain 17\% C16 in CAP; CAP-mc-C8 contains 20\% mc and 17\% C8 in CAP; CAP-mc-C16 contains $20 \%$ mc and $17 \%$ C16 in CAP and Cref is PLA-based material with 20\% cellulose fibre.

The tensile strength results in Table 5 and Figure 7 are in general reflecting the challenges between printed layer adhesion and pores by showing that during tensile stress the breakage of the sample occurs earlier than with injection molded samples. The strength results of printed samples are $10 \%$ to $78 \%$ lower than those of injection molded samples. The strength reduction in 3D printed and mc containing samples is the highest being $49 \%$ and $78 \%$ in CP-mc-C16 and CAP-mc-C16 respectively. Even in Cref the reduction is $56 \%$ from $26.6 \mathrm{MPa}$ of injection molded to $11.6 \mathrm{MPa}$ of $3 \mathrm{D}$ printed samples. The neat commercial polymer $\mathrm{CP}$ retained the strength best also in printed structure having the tensile strength value $23.6 \mathrm{MPa}$ in printed samples and $26 \mathrm{MPa}$ in injection molded samples, which is in-line with the relatively good adhesion between printed layers visible also in Figure 5. The low strength properties and porosity of 3D printed wood filled composite, but also their relation to printing parameters are reported also by Le Duigou et al. [37]. The best strength values for mc containing 3D printed material were obtained for CP-mc-C16, 12.9 MPa.

When focusing on injection molded samples, we can see that the tensile strength of all the $20-21 \%$ mc containing materials are at the level of 24 to $32 \mathrm{MPa}$. Even though CAP-C8 had some miscibility challenges, visible in Figure 4, by showing tensile strength $10.9 \mathrm{MPa}$, the $21 \%$ mc addition increased the strength value to $31.8 \mathrm{MPa}$, which may come from the partial impregnation of $\mathrm{C} 8$ to $\mathrm{mc}$. However, the bigger miscibility challenges, visible in Figure 4, with C16 in CAP-C16 were not reflected to strength values of injection molded samples; that had tensile strength of $29 \mathrm{MPa}$ but reduced to $24 \mathrm{MPa}$ with mc addition.

The plasticization effect of $\mathrm{C} 8$ and $\mathrm{C} 16$ can be seen when comparing the tensile strength results of $\mathrm{CP}-\mathrm{mc}, \mathrm{CP}-\mathrm{mc}-\mathrm{C} 8$ and $\mathrm{CP}-\mathrm{mc}-\mathrm{C} 16$ having the strength values $28.9 \mathrm{MPa}, 26.0 \mathrm{MPa}$ and 25.5 MPa respectively.

The results for tensile modulus (Young's) in Table 5 and Figure 8 are reflecting the same layer adhesion and porosity challenges as tensile strength results for $3 \mathrm{D}$ printed samples, as compared to injection molded, by showing up to $50 \%$ lower values for 3D printed fiber containing samples. The highest reduction is with CP-mc-C8 from $1670 \mathrm{MPa}$ to $837 \mathrm{MPa}$. Modulus of the neat commercial polymer $\mathrm{CP}$ was in the same level with both manufacturing methods, if standard deviation is considered, $1360 \mathrm{MPa}$ and $1297 \mathrm{MPa}$ for injection molded and 3D printed respectively, provided by the good layer adhesion. 
In injection molded samples the mc addition to $\mathrm{CP}$ increases modulus in $\mathrm{CP}$-mc by $26 \%$ from $1360 \mathrm{MPa}$ to $1710 \mathrm{MPa}$. $4 \% \mathrm{C} 8$ addition retained the modulus at the same level being $1670 \mathrm{MPa}$ in CP-mc-C8, but $4 \%$ cellulose-ester C16 increased the modulus to $1840 \mathrm{MPa}$, which may be partially due to increased fiber dispersion. The modulus was also increased in CAP-based compounds after mc addition. In 17\% C8 containing materials the mc addition caused $32 \%$ increase in modulus from $1539 \mathrm{MPa}$ to $2034 \mathrm{MPa}$ and in $17 \% \mathrm{C} 16$ containing materials $18 \%$ increase from $1680 \mathrm{MPa}$ to $1981 \mathrm{MPa}$. This modulus increase due to fiber material addition is in-line with previous studies [18].

The modulus of commercial PLA-based Cref with $20 \%$ fiber was $2642 \mathrm{MPa}$, thus being the stiffest in injection molded material, but still having the modulus reduction similar with the cellulose polymer-based material as 3D printed, when the modulus was 34\% lower, $1754 \mathrm{MPa}$. Even though the layer adhesion, visible in Figure 5 was good, the 3D printed material contained quite a lot of porosity.

The elongation values for injection molded pure cellulose $\mathrm{C} 16$ and $\mathrm{C} 8$ are reported to be $12 \%$ and $50 \%$, respectively [16]. These values are much higher than reported for commercially available shorter chain-length CAP [38]. By combining C8 and C16 with $\mathrm{CAP}$ and $\mathrm{CP}$ was assumed to achieve materials with higher elongation properties, which did not come true with these compounds.

The strain at yield value for CP is quite same, $3.5-4 \%$, for both injection molded and 3D printed samples due to good layer adhesion. The $21 \% \mathrm{mc}$ and $4 \%$ cellulose-ester addition reduced the strain by dropping the strain value to level of $2.8-3.0 \%$. C16 seems to have a slightly bigger reduction effect than $\mathrm{C} 8$. In 3D printed CP-based materials the reduction in strain was highest in $\mathrm{CP}-\mathrm{mc}-\mathrm{C} 8$ to $0.9 \%$ that may indicate fiber dispersion challenges. In CP-mc and CP-mc-C16, with strain $2.5 \%$, fiber dispersion is probably good reflecting material internal elongation during stress.

In CAP-based injection molded samples, the strain in $17 \% \mathrm{C} 16$ containing material was $2.2 \%$ and clearly higher than in $17 \% \mathrm{C} 8$ material, $0.8 \%$, even though the elongation of $\mathrm{C} 8$ is reported to be higher. The difference may be due to some miscibility challenges between cellulose esters ( $\mathrm{C} 8$ and C16) and CAP. C8 may be better compatible with CAP, when $\mathrm{mc}$ is added, which can be seen as strain at yield value for CAP-mc-C8, 2.1\%. The 3D printed CAP-based materials showed all poor strain values due to poor layer adhesion as explained earlier. The strain at yield in 3D printed Cref was $22 \%$ lower than in injection molded material, $2.1 \%$ vs. $1.64 \%$, indicating good fiber dispersion and quite good layer adhesion.

\subsubsection{Charpy Impact Strength}

Charpy impact strength results for injection molded and 3D printed samples are presented in Table 6 and Figure 10. All the other results are for unnotched samples except $\mathrm{CP}$, which did not break as unnotched and the test was made also as notched.

The Charpy impact strength (unnotched) results in Table 6 and Figure 10 are reflecting the brittle nature of the $3 \mathrm{D}$ printed mc containing commercial polymer $\mathrm{CP}$ containing samples, but also that of PLA-based cellulose fiber containing samples. As such the CP is a very tough material, which cannot be broken as unnotched, so the result is presented for notched samples. The high standard deviation in 3D printed sample is maybe due to the fact, that during notch preparation some detachment between printed layers may have occurred.

The mc addition drops the impact strength result of the injection molded samples to the level of $21-22 \mathrm{~kJ} / \mathrm{m}^{2}$ and $3 \mathrm{D}$ printed results to $5-6 \mathrm{~kJ} / \mathrm{m}^{2}$ with or without $4 \%$ celluloseester (C8 or C16) addition. With CAP the $17 \%$ addition of cellulose-esters shows differences between $\mathrm{C} 8$ and $\mathrm{C} 16$ so that $\mathrm{C} 16$ is providing more impact ductility to the material than $\mathrm{C} 8$, which can be seen by comparing the results for injection molded samples CAP-C8, $4.5 \mathrm{~kJ} / \mathrm{m}^{2}$, and CAP-C16, $15.2 \mathrm{~kJ} / \mathrm{m}^{2}$. The impact strength of 3D printed samples from these two materials without fillers are surprisingly high when considering the loose layer adhesion visible in Figure 5, $8.4 \mathrm{~kJ} / \mathrm{m}^{2}$ for CAP-C 8 and $16 \mathrm{~kJ} / \mathrm{m}^{2}$ for CAP-C16. However, 
during the test, the impact force needs to transfer from individual 3D printed string to another via only small contacts and not to transfer inside the even material, which may explain the impact results being even higher than for injection molded ones.

The mc addition to CAP containing 17\% C16 dropped down the impact strength of injection molded material by $49 \%$ to $7.8 \mathrm{~kJ} / \mathrm{m}^{2}$. With $17 \% \mathrm{C} 8$ and mc the impact strength values were retained despite of the mc addition or even increased from $4.5 \mathrm{~kJ} / \mathrm{m}^{2}$ to $6.8 \mathrm{~kJ} / \mathrm{m}^{2}$. Also, in 3D printed samples with high cellulose-ester content $(17 \%)$ and $\mathrm{mc}$ the impact strength was retained in the level of injection molded materials. That may also be due to similar force transfer effects between printed layers than in materials without mc.

The Charpy impact strength of injection molded PLA-based Cref was $18.8 \mathrm{~kJ} / \mathrm{m}^{2}$, thus being better than with CAP-based materials, but 10-15\% lower than in CP-based materials. As $3 \mathrm{D}$ printed the impact strength of Cref was dropped to $7.7 \mathrm{~kJ} / \mathrm{m}^{2}$, indicating quite good layer adhesion, but high porosity and probably challenges in fiber polymer adhesion, which were also visible as gaps between fiber and polymer in Figure 4 .

Table 6. Charpy impact strength results for injection molded and 3D printed materials. Unnotched samples except $\mathrm{CP}$, which did not break as unnotched.

\begin{tabular}{|c|c|c|}
\hline Code & Injection Moulded & 3D Printed \\
\hline & $\mathrm{kJ} / \mathrm{m}^{2}$ & $\mathrm{~kJ} / \mathrm{m}^{2}$ \\
\hline $\mathrm{CP}$ & $\begin{array}{c}\text { no break unnotched } \\
20.1 \pm 0.7 \text { notched }\end{array}$ & $\begin{array}{l}\text { no break unnotched } \\
26.0 \pm 19.0 \text { notched }\end{array}$ \\
\hline $\mathrm{CP}-\mathrm{mc}$ & $21.9 \pm 1.1$ & $5.1 \pm 0.8$ \\
\hline $\mathrm{CP}-\mathrm{mc}-\mathrm{C} 8$ & $22.5 \pm 1.6$ & $6.0 \pm 0.7$ \\
\hline $\mathrm{CP}-\mathrm{mc}-\mathrm{C} 16$ & $20.9 \pm 2.7$ & $5.1 \pm 0.3$ \\
\hline CAP-C8 & $4.5 \pm 0.7$ & $8.4 \pm 2.5$ \\
\hline CAP-C16 & $15.2 \pm 5.0$ & $16 \pm 3.0$ \\
\hline CAP-mc-C8 & $6.8 \pm 0.6$ & $8.3 \pm 4.3$ \\
\hline CAP-mc-C16 & $7.8 \pm 2.0$ & $7.2 \pm 0.9$ \\
\hline Cref & $18.8 \pm 2.0$ & $7.7 \pm 1.2$ \\
\hline
\end{tabular}

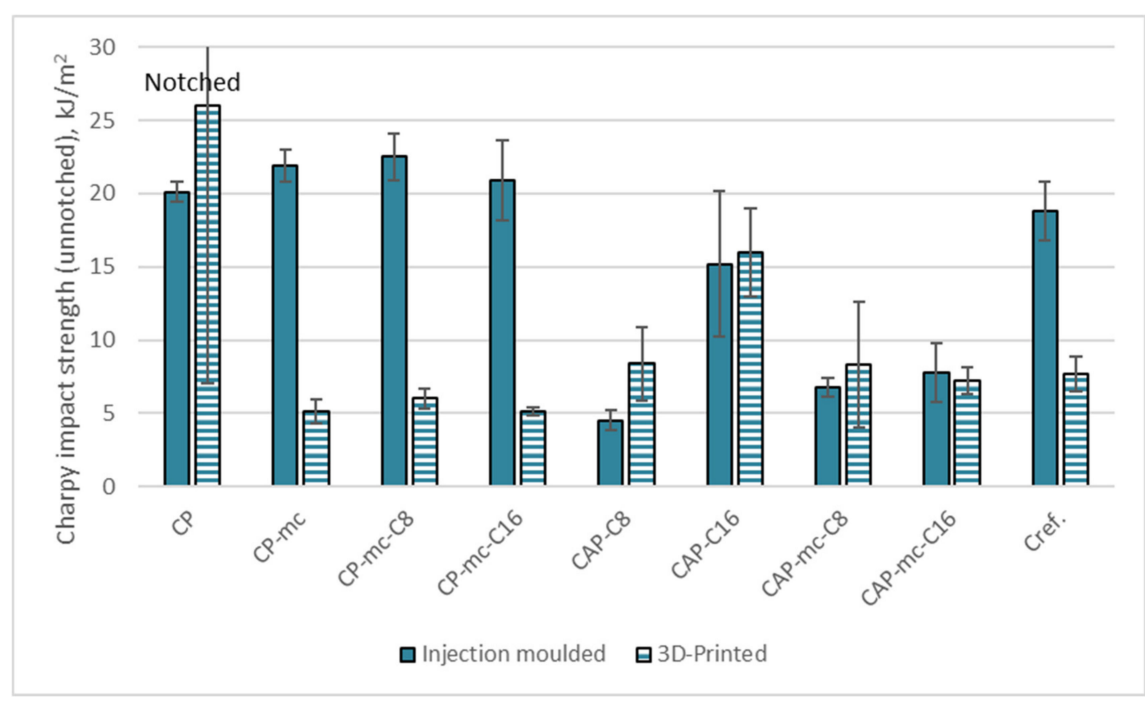

Figure 10. Charpy impact strength results for injection molded and $3 \mathrm{D}$ printed materials. Unnotched samples except $\mathrm{CP}$, which did not break as unnotched. $\mathrm{CP}$ is commercial cellulose acetate propionate with $13 \%$ commercial plasticizer; $\mathrm{CP}$-mc is $20 \%$ microcellulose containing CP; CP-mc-C8 contains also $4 \%$ cellulose octanoate C8; CP-mc-C16 contains $4 \%$ of cellulose palmitate $\mathrm{C} 16$; CAP-C8 contains neat cellulose acetate propionate (CAP) with 17\% C8; CAP-C16 contain $17 \%$ C16 in CAP; CAP-mc-C 8 contains $20 \%$ mc and $17 \%$ C 8 in CAP; CAP-mc-C16 contains $20 \%$ mc and $17 \%$ C16 in CAP and Cref is PLA-based material with $20 \%$ cellulose fibre. 


\section{Materials and Methods}

3.1. Materials

\subsubsection{Materials in Production of Thermoplastic Cellulose Additive}

The cellulose for production of thermoplastic cellulose additives was commercial softwood dissolving grade pulp (Domsjö Fabriker AB, Sweden) with average Mw 520 kDa. The pulp was ozone pretreated according to a method described by Willberg-Keyriläinen et al. [35] to reduce the molar mass to $84 \mathrm{kDa}$. All other reagents were analytical grade and purchased from Sigma-Aldrich (Merck KGaA, Dramstadt, Germany).

\subsubsection{Materials in Manufacturing of Cellulose-Based Compound}

Polymer matrices used for preparation of cellulose based compounds were cellulose acetate propionate without plasticizer (CAP) from Sigma-Aldrich (Merck KGaA, Darmstadt, Germany) with average Mn $\sim 75,000$, acetyl content $2.5 \mathrm{w}-\%$ and propionyl content $46 \mathrm{wt}-$ $\%$, and cellulose acetate propionate CELLIDOR CP300-13 (CP) (Albis Plastics GmbH, Hamburg, Germany) with phthalate free plasticizer content of $13 \%$ and melt flow rate $7.5 \mathrm{~cm}^{3} / 10 \mathrm{~min}\left(210^{\circ} \mathrm{C}, 2.16 \mathrm{~kg}\right)[39,40]$. Cellulose fiber used in compounds in amount of 20 wt- $\%$ was microcrystalline cellulose VIVAPUR 105 (JRS Pharma GmbH, Weissenborn, Germany) with average particle size by Laser diffraction $15 \mu \mathrm{m}$. In coupling of fiber and polymer was used reactive epoxidized linseed oil Lankroflex ${ }^{\mathrm{TM}} \mathrm{L}$ (Valtris Specialty Chemicals, Independence, Ohio, USA). As additional plasticizer in compound in amounts of $4 \mathrm{wt}-\%$ and $\mathrm{wt}-17 \%$, a thermoplastic cellulose-esters prepared at VTT according to method explained in chapter 3.2.1, were used. As commercial 3D printable reference material was used PLA-based compound containing 20\% cellulose fiber (Cref).

\subsection{Methods}

\subsubsection{Preparation of Thermoplastic Cellulose Additive}

Thermoplastic cellulose additives (cellulose octanoate (C8) and cellulose palmitate (C16) were prepared using the homogeneous method presented by Willberg-Keyriläinen et al. [35,41]. In this method, dry cellulose was first dissolved in a 5\% $\mathrm{LiCl} / \mathrm{DMAc}$ solution. Then fatty acid chloride (octanoyl chloride (C8) or palmitoyl chloride (C16), 3-4 equivalents to cellulose anhydroglucose unit (AGU) was added to the cellulose mixture using pyridine (3.6-4.6 equivalents/AGU) as catalyst. The reaction temperature was $80^{\circ} \mathrm{C}$ and reaction time 16 hours. The cellulose esters were precipitated and washed with ethanol, C16 ester was additionally washed with acetone. The purity of these esters was determined using FTIR (Fourier-transform infrared spectroscopy) and ${ }^{1} \mathrm{H}$ NMR (Nuclear Magnetic Resonance Spectroscopy) analyses, and no residues of free acid or fatty acid ethyl ester were detected after appropriate washing.

The degree of substitution (DS) of the prepared thermoplastic cellulose additives was analyzed using the solid state nuclear magnetic resonance (600 MHz NMR spectrometer, 10,000 scans, 10s recycle time, Agilent Technologies, Santa Clara, CA, USA) by comparing the cellulose esters carbonyl carbon $(175 \mathrm{ppm})$ integrals with the cellulose $\mathrm{C} 1$ signal (105 ppm) integral. According to the NMR results, the DS values of thermoplastic cellulose C8 and cellulose C16 were 1.1 and 1.0, respectively.

\subsubsection{Processing of Thermoplastic Materials}

Before compounding a coupling agent Lankroflex L was mixed with mc in amount of $5 \%$ in relation to mc dry weight using a blade blender and the mixture was dried in $50{ }^{\circ} \mathrm{C}$ overnight. Also, thermoplastic cellulose additive $\mathrm{C} 8$ or $\mathrm{C} 16$ was dried in $50^{\circ} \mathrm{C}$ overnight before compounding. The polymer matrices CAP and Cellidor CP300-13 (CP) were dried in $80^{\circ} \mathrm{C}$ for $2 \mathrm{~h}$.

Additive (Lankroflex L) containing mc and cellulose-ester were compounded with polymer matrices (CAP or CP-13) according to amounts presented in Table 1 using a corotating twin-screw extruder (Berstorff ZE 25x33 D, Berstorff GmbH, Hanover, Germany). The extruder zone temperatures ranged from $80^{\circ} \mathrm{C}$ to $205^{\circ} \mathrm{C}$, speed $100 \mathrm{rpm}$ and output 
$2 \mathrm{~kg} / \mathrm{h}$. The small pellets or granules from compounding were further processed using injection molding and 3D printing using granular printer explained in Section 3.2.3.

After compounding, the samples were injection molded with an injection-molding machine (Engel ES 200/50 HL, Engel Maschinenbau Geschellschaft m.b.H, Schwefberg, Austria) to test specimens according to ISO 527. The processing temperatures during injection molding were from 180 to $200{ }^{\circ} \mathrm{C}$ in the screw and $205^{\circ} \mathrm{C}$ at nozzle and mold temperature $70^{\circ} \mathrm{C}$. The injection molding of Cref succeeded also in those temperatures.

\subsubsection{Additive Manufacturing}

Samples for tensile and impact strength (see Section 3.2.6) testing were prepared using fused granular fabrication (FGF) AM system delivered by Brinter ${ }^{\circledR}$. The system comprises a standard heated glass printing bed, tailored printing head for thermoplastic granules and system's own software to control the movement of the printing head. BuildTak ${ }^{\mathrm{TM}} 3 \mathrm{D}$ printing sheets and glue stick were used on the top of the heated bed in order to increase the adhesion of the first layers.

Based on preliminary 3D printing trials, a printing speed of $15 \mathrm{~mm} / \mathrm{s}$ and brass nozzles, type E3D-V6, with a diameter of $0.8 \mathrm{~mm}$ were selected and used in manufacturing of all samples. All samples were manufactured horizontally using a concentric infill pattern and $100 \%$ infill percentage. The number of layers were 4 and 8 for tensile and impact strength samples, respectively. The only exception was CAP-mc-C8 composition that had a very limited $3 \mathrm{D}$ printability and due to it the layer height of $0.25 \mathrm{~mm}$ was used and the number of layers was therefore double compared with the other material compositions. Temperatures of the nozzle and the bed and layer heights used in the manufacturing of the samples are collected in Table 7.

Table 7. Parameters used in 3D printing trials.

\begin{tabular}{ccc}
\hline Code & $\begin{array}{c}\text { Temperature } \\
\text { Nozzle/Bed } \\
\left({ }^{\circ} \mathbf{C}\right)\end{array}$ & Layer Height $(\mathbf{m m})$ \\
\hline CP & $200 / 60$ & 0.5 \\
\hline CP-mc & $215 / 60$ & 0.5 \\
\hline CP-mc-C8 & $210 / 60$ & 0.5 \\
\hline CP-mc-C16 & $219 / 60$ & 0.5 \\
\hline CAP-C8 & $185 / 60$ & 0.5 \\
\hline CAP-C16 & $220 / 60$ & 0.5 \\
\hline CAP-mc-C8 & $220 / 60$ & 0.25 \\
\hline CAP-mc-C16 & $224 / 60$ & 0.5 \\
\hline Cref & $215 / 60$ & 0.5 \\
\hline
\end{tabular}

\subsubsection{Thermal Analytics}

Thermo-gravimetric analytics (TGA) were performed using NETZSCH STA449F1 analyzer (NEZSCH GmbH, Selb, Germany). The TGA measurements were carried out in air atmosphere from 40 to $800{ }^{\circ} \mathrm{C}$ with a heating rate of $10^{\circ} \mathrm{C} / \mathrm{min}$.

DSC (Differential scanning chromatography) analysis for compounds was made using NETZSCH DSC 204F1 Phoenix 240-12-0287-L (NEZSCH GmbH, Selb, Germany). The heating profile was two cycles at with $10^{\circ} \mathrm{C} / \mathrm{min}$ heating and the cooling speed from $-20{ }^{\circ} \mathrm{C}$ to $220^{\circ} \mathrm{C}$ and back to $-20^{\circ} \mathrm{C}$.

\subsubsection{Scanning Electron Microscopy (SEM)}

The morphology of the injection molded, and 3D printed samples was studied using a SEM (scanning electron microscopy) from a cross-cut section of the test bar. The samples 
were cooled in liquid nitrogen and broken to two. The analysis was made from the broken surface. The sample surface was coated with gold to prevent surface charging. Gold film thickness on the sample surface was 50-70 nm. Analyses were made using JEOL JSM T100 (JEOL ltd., Tokyo, Japan) with a voltage of $25 \mathrm{kV}$.

\subsubsection{Mechanical Tests}

Tensile tests were performed according to ISO-527 standard using an Instron 4505 Universal Tensile Tester (Instron Corp., Canton, MA, USA) and an Instron 2665 Series High Resolution Digital Automatic Extensometer (Instron Corp., Canton, MA, USA) with a $10 \mathrm{kN}$ load cell and a $5 \mathrm{~mm} / \mathrm{min}$ cross-head speed. Five parallel specimens were tested for each sample material to obtain the average values of the tensile properties.

Impact strengths were determined according to ISO-179 standard using a Charpy Ceast Resil 5.5 Impact Strength Machine (CEAST S.p.a., Torino, Italy). Charpy impact strength tests in edgewise orientation was applied to the unnotched specimens. Impact testing was made for six to ten replicates to obtain an average value and variance.

The test specimens were kept in standard conditions $\left(23^{\circ} \mathrm{C}, 50 \%\right.$ relative humidity) for at least five days before testing.

\subsubsection{Heat Distortion Temperature (HDT)}

Heat distortion temperature (HDT) was measured according to the ISO-75 standard using method A with 1.8. MPa stress on the sample. HDT was determined by using the Ceast HDT 3 VICAT P/N 6911.000 (Ceast S.p.a., Torino, Italy) and for three parallel samples.

\section{Conclusions}

The aim of this work was to study the properties of thermoplastic cellulose-based composites and how they could be applied on a novel 3D printing technique, printing of granules. The focus was to maximize the cellulose content of the composite by using two cellulose-based polymer matrices; neat cellulose acetate propionate (CAP) polymer and commercial cellulose acetate propionate containing 13\% commercial plasticizer (CP), two novel cellulose-ester additives and microcellulose $(\mathrm{mc})$ to bring improved stiffness to the composite material. The novel cellulose-ester additives were cellulose octanoate (C8) and cellulose-palmitate (C16), and their function as fiber dispersing and CAP plasticizer additive was analyzed. To evaluate the property level of the developed cellulose-based composites compared to existing commercial materials a commercial PLA-based cellulose fiber containing composite material was used as a reference (Cref). Materials were also injection molded to see their best possible material properties.

It was possible to manufacture all the materials for test samples using 3D granular printer, however this manufacturing method revealed clearly the differences between the material combinations. The commercial $\mathrm{CP}$, containing $13 \%$ commercial plasticizer, was as such printable and showed good strength properties both as 3D printed and injection molded. Also, CP-based, mc containing composite was printable with and without $4 \%$ cellulose-ester addition and showed adequate adhesion between the printed layers, but also high porosity inside the material. The more experimental, CAP-based materials with $17 \%$ cellulose-esters had challenges in printed layer adhesion and the surface of compounds were oily indicating that the amount of cellulose esters, $17 \%$, was too high to mix properly with CAP. Also, the mc-containing CAP compounds showed high porosity inside the material in 3D printed samples. Porosity was discovered also in the 3D printed reference PLA-based Cref. The high porosity reflects the challenges within granular printing method. However, the main objective of this work was to compare different material compositions and to manufacture samples using 3D printing for further analysis and testing. For that reason, most of the 3D printing parameters were kept unchanged after a careful pre-testing of those. We believe that the properties of the 3D printed samples can be further enhanced by optimization of the 3D printing parameters for the selected material in the future. 
The tensile strength properties of the injection molded CP-materials and Cref were comparable to each other having tensile strength between 25.5 to $29 \mathrm{MPa}$. The Charpy impact strength in CP-compounds was $12-20 \%$ higher compared to Cref, $18.8 \mathrm{~kJ} / \mathrm{m}^{2}$. However, the tensile modulus of $\mathrm{Cref}$ is $30-37 \%$ higher compared to $\mathrm{CP}$-compounds, $\mathrm{CP}$ mc-C8 had modulus of $1670 \mathrm{MPa}, \mathrm{CP}-\mathrm{mc} 1720 \mathrm{MPa}$ and CP-mc-C16 $1840 \mathrm{MPa}$. Regarding the CAP-mc compounds, there is potential for higher tensile strength and modulus with C8 addition. CAP-mc-C8 had tensile strength of $31.8 \mathrm{MPa}$ and modulus of $2034 \mathrm{MPa}$, but quite low impact strength, $6.8 \mathrm{~kJ} / \mathrm{m}^{2}$, thus showing the brittleness of the material. The cellulose-ester, $\mathrm{C} 8$ and $\mathrm{C} 16$, materials showed some miscibility challenges with CAP, especially in high contents, $17 \%$, which seemed to be too much. However, there was indication of improved fiber (mc) dispersion when lower cellulose-ester amounts, $4 \%$, were used.

The thermal behavior of the PLA-based Cref showed very constant $\mathrm{Tg}$ of $60^{\circ} \mathrm{C}$ in two heating cycles and HDT temperature $55.4^{\circ} \mathrm{C}$. The commercial CP-based materials stabilized after the first heating cycle in DSC-analysis showing the Tg of $95-103{ }^{\circ} \mathrm{C}$ during the second heating, depending on the compound. CP-mc-C16 had the highest HDT-value, $62.1^{\circ} \mathrm{C}$, of the CP-based materials but with CAP and C16 (17\%) as the only additive, HDT-value of $82.1^{\circ} \mathrm{C}$ was achieved.

This study indicates a potential to tailor high temperature resistant cellulose composite materials using thermoplastic cellulose acetate propionate as the polymer matrix material in combination with microcellulose and possibly with small amounts of novel cellulose-esters as fiber dispersing additive. More studies are still needed to optimize the additive and filler amounts, as well as cellulosic polymer base towards all-cellulose composites competitive with PLA-based commercial composites. Also, optimization for printing parameters is needed, if the focus is $3 \mathrm{D}$ printable materials and future manufacturing technologies.

Author Contributions: Writing-original draft preparation, K.I.; writing-3D printing related parts, S.M.-K.; writing-3D printing results, A.N.; cellulose-ester preparation and writing-cellulose-ester related issues, P.W.-K.; writing-cellulose related issues, J.R.; review, O.-V.K.; review and project administration, H.K. All authors have read and agreed to the published version of the manuscript.

Funding: This research was funded by EU-project NOVUM, grant number 768604.

Institutional Review Board Statement: Not applicable.

Informed Consent Statement: Not applicable.

Data Availability Statement: Not applicable.

Acknowledgments: The authors would like to thank Sini-Tuuli Rauta, Petri Kuusipalo, Olli Linjamaa and Amelié Tribot for material processing and contribution in sample analytics.

Conflicts of Interest: The authors declare no conflict of interest.

Sample availability: Samples of the compounds containing microcellulose are available from the authors.

\section{References}

1. AMFG. Available online: https://amfg.ai/industrial-applications-of-3d-printing-the-ultimate-guide/ (accessed on 12 February 2021).

2. Kellens, K.; Baumers, M.; Gutowski, T.G.; Flanagan, W.; Lifset, R.; Duflou, J.R. Environmental Dimensions of Additive Manufacturing: Mapping Application Domains and Their Environmental Implications. J. Ind. Ecol. 2017, 21, S49-S68. [CrossRef]

3. European Commission. Available online: https:/ / ec.europa.eu/info/policies/climate-action_en (accessed on 12 February 2021).

4. Ellen Macarthur Foundation. Available online: https://www.ellenmacarthurfoundation.org/assets/downloads/publications/ NPEC-Hybrid_English_22-11-17_Digital.pdf (accessed on 12 February 2021).

5. Campbell, I.; Bourell, D.; Gibson, I. Additive manufacturing: Rapid prototyping comes of age. Rapid Prototyp. J. 2012, 18, 255-258. [CrossRef]

6. SFS-EN ISO/ASTM 52900:2017. Additive manufacturing-General principles-Terminology; Suomen Standardisoimisliitto: Helsinki, Finland, 2017. 
7. Woern, A.; Byard, D.; Oakley, R.; Fiedler, M.; Snabes, S.; Pearce, J. Fused Particle Fabrication 3-D Printing: Recycled Materials' Optimization and Mechanical Properties. Materials 2018, 11, 1413. [CrossRef] [PubMed]

8. Cincinnati. Available online: https://www.e-ci.com/3d-scoop/2020/9/17/pellet-vs-filament-3d-printing (accessed on 12 February 2021).

9. Fabbaloo. Available online: https://www.fabbaloo.com/blog/2018/5/10/the-other-reason-for-3d-printing-pellets (accessed on 12 February 2021).

10. Klemm, D.; Philipp, B.; Heinze, T.; Heinze, U.; Wagenknecht, W. Comprehensive Cellulose Chemistry; Wiley-VCH Verlag GmbH \& Co. KGaA: Weinheim, Germany, 1998; Volume 1, ISBN 3527294139.

11. Edgar, K.J.; Buchanan, C.; Debenham, J.; Rundquist, P.; Seiler, B.; Shelton, M.; Tindall, D. Advances in cellulose ester performance and application. Prog. Polym. Sci. 2001, 26, 1605-1688. [CrossRef]

12. Klemm, D.; Heublein, B.; Fink, H.-P.; Bohn, A. Cellulose: Fascinating biopolymer and sustainable raw material. Angew. Chemie Int. Ed. 2005, 44, 3358-3393. [CrossRef]

13. Luan, Y.; Wu, J.; Zhan, M.; Zhang, J.; Zhang, J.; He, J. “One pot” homogeneous synthesis of thermoplastic cellulose acetate-graftpoly(l-lactide) copolymers from unmodified cellulose. Cellul. 2013, 20, 327-337. [CrossRef]

14. Havimo, M.; Jalomäki, J.; Granström, M.; Rissanen, A.; Iivanainen, T.; Kemell, M.; Heikkilä, M.; Sipi, M.; Kilpeläinen, I. Mechanical strength and water resistance of paperboard coated with long chain cellulose esters. Packag. Technol. Sci. 2011, 24, 249-258. [CrossRef]

15. Crépy, L.; Chaveriat, L.; Banoub, J.; Martin, P.; Joly, N. Synthesis of cellulose fatty esters as plastics-influence of the degree of substitution and the fatty chain length on mechanical properties. ChemSusChem 2009, 2, 165-170. [CrossRef]

16. Willberg-Keyriläinen, P.; Orelma, H.; Ropponen, J. Injection Molding of Thermoplastic Cellulose Esters and Their Compatibility with Poly(Lactic Acid) and Polyethylene. Materials 2018, 11, 2358. [CrossRef] [PubMed]

17. Willberg-Keyriläinen, P.; Rokkonen, T.; Malm, T.; Harlin, A.; Ropponen, J. Melt spinnability of long chain cellulose esters. J. Appl. Polym. Sci. 2020, 49588. [CrossRef]

18. Pickering, K.L.; Efendy, M.G.A.; Le, T.M. A review of recent developments in natural fibre composites and their mechanical performance. Compos. Part.Part A Appl. Sci. Manuf. 2016, 83, 98-112. [CrossRef]

19. Nath, S.D.; Nilufar, S. An Overview of Additive Manufacturing of Polymers and Associated Composites. Polym. 2020, 12, 2719. [CrossRef]

20. Calì, M.; Pascoletti, G.; Gaeta, M.; Milazzo, G.; Ambu, R. A New Generation of Bio-Composite Thermoplastic Filaments for a More Sustainable Design of Parts Manufactured by FDM. Appl. Sci. 2020, 10, 5852. [CrossRef]

21. UPM Formi 3D-Printing Products. Available online: https://www.upmformi.com/biocomposite-products/3d-printing/ (accessed on 16 February 2021).

22. ColorFabb Filled Filaments. Available online: https://colorfabb.com/filaments/special-filaments (accessed on 16 February 2021).

23. Mohan, D.; Teong, Z.K.; Bakir, A.N.; Sajab, M.S.; Kaco, H. Extending cellulose-based polymers application in additive manufacturing technology: A review of recent approaches. Polym. 2020, 12, 1876. [CrossRef] [PubMed]

24. Wang, Q.; Sun, J.; Yao, Q.; Ji, C.; Liu, J.; Zhu, Q. 3D printing with cellulose materials. Cellulose 2018, 25, 4275-4301. [CrossRef]

25. Martikka, O.; Kärki, T.; Wu, Q. Mechanical properties of 3D-printed wood-plastic composites. Key Eng. Mater. 2018, 777, 499-507. [CrossRef]

26. Mazzanti, V.; Malagutti, L.; Mollica, F. FDM 3D Printing of Polymers Containing Natural Fillers: A Review of their Mechanical Properties. Polym. 2019, 11, 1094. [CrossRef]

27. Khan, M.Z.R.; Srivastava, S.K.; Gupta, M.K. A state-of-the-art review on particulate wood polymer composites: Processing, properties and applications. Polym. Test. 2020, 89, 106721. [CrossRef]

28. Karakoç, A.; Rastogi, V.K.; Isoaho, T.; Tardy, B.; Paltakari, J.; Rojas, O.J. Comparative screening of the structural and thermomechanical properties of FDM filaments comprising thermoplastics loaded with cellulose, carbon and glass fibers. Materials 2020, 13. [CrossRef]

29. Farah, S.; Anderson, D.G.; Langer, R. Physical and mechanical properties of PLA, and their functions in widespread applicationsA comprehensive review. Adv. Drug Deliv. Rev. 2016, 107, 367-392. [CrossRef]

30. Teramoto, Y. Functional Thermoplastic Materials from Derivatives of Cellulose and Related Structural Polysaccharides. Molecules 2015, 20, 5487-5527. [CrossRef]

31. Böhler, S.; Bartel, M.; Bohn, A.; Jacob, R.; Ganster, J.; Büsse, T.; Balko, J. Highly dense cellulose acetate specimens with superior mechanical properties produced by fused filament fabrication. Polym. 2020, 194, 122388. [CrossRef]

32. Azad, M.A.; Olawuni, D.; Kimbell, G.; Badruddoza, A.Z.M.; Hossain, M.S.; Sultana, T. Polymers for Extrusion-Based 3D Printing of Pharmaceuticals: A Holistic Materials-Process Perspective. Pharm. 2020, 12, 124. [CrossRef] [PubMed]

33. Mervine, N.; Brätt, K.; Saloni, D. A Review of Sustainable Materials Used in Thermoplastic Extrusion and Powder Bed Melting Additive Manufacturing. In Proceedings of the Advances in Intelligent Systems and Computing; Volume 1216 AISC; Springer International Publishing: Cham, Switzerland, 2020; pp. 95-102.

34. Lamm, M.E.; Wang, L.; Kishore, V.; Tekinalp, H.; Kunc, V.; Wang, J.; Gardner, D.J.; Ozcan, S. Material extrusion additive manufacturing of wood and lignocellulosic filled composites. Polymers 2020, 12, 2115. [CrossRef] [PubMed]

35. Willberg-Keyriläinen, P.; Asikainen, S.; Harlin, A.; Talja, R.; Ropponen, J. The effect of cellulose molar mass on the properties of palmitate esters. Carbohydr. Polym. 2016, 151, 988-995. [CrossRef] 
36. Duchatel-Crépy, L.; Joly, N.; Martin, P.; Marin, A.; Tahon, J.-F.; Lefebvre, J.-M.; Gaucher, V. Substitution degree and fatty chain length influence on structure and properties of fatty acid cellulose esters. Carbohydr. Polym. 2020, 234, 115912. [CrossRef]

37. Le Duigou, A.; Castro, M.; Bevan, R.; Martin, N. 3D printing of wood fibre biocomposites: From mechanical to actuation functionality. Mater. Des. 2016, 96, 106-114. [CrossRef]

38. Rynkowska, E.; Fatyeyeva, K.; Kujawa, J.; Dzieszkowski, K.; Wolan, A.; Kujawski, W. The effect of reactive ionic liquid or plasticizer incorporation on the physicochemical and transport properties of cellulose acetate propionate-based membranes. Polym. 2018, 10, 86. [CrossRef] [PubMed]

39. Merck. Cellulose Acetate Propionate Product Information. Available online: https://www.sigmaaldrich.com/catalog/product/ aldrich/330183?lang=fi\&region=FI (accessed on 16 February 2021).

40. Albis Plastics GmbH Material Data Center Datasheet for Cellidor CP300-13. Available online: https:/ / www.materialdatacenter. $\mathrm{com} / \mathrm{ms} / \mathrm{en} /$ Cellidor/ALBIS+PLASTIC+GmbH/10 (accessed on 16 February 2021).

41. Willberg-Keyriläinen, P.; Vartiainen, J.; Harlin, A.; Ropponen, J. The effect of side-chain length of cellulose fatty acid esters on their thermal, barrier and mechanical properties. Cellulose 2017, 24, 505-517. [CrossRef] 